Partial Differential Equations - Mixed type, nonlinear systems in polygonal domains, by V. A. Solonnikov and M. A. Vivaldi, communicated on 9 November 2012.

\title{
In Memoriam Gaetano Fichera
}

\begin{abstract}
We prove existence, uniqueness results and coercive estimates in the weighted Sobolev spaces for a linear problem of mixed type in a bounded domain $\Omega \subset \mathbb{R}^{2}$ whose boundary is smooth everywhere except a single angular point $x=0$ with the aperture of the angle $\theta>\pi$. In addition, we establish a stability result for a non-linear system of mixed type. The results of the paper and the proofs extend to the case of polygonal domains.
\end{abstract}

Key WORDS: Non-linear systems of mixed type in irregular domains, estimates in weighted Sobolev spaces, stability results.

Mathematics Subject Classification: $35 \mathrm{~K} 20$, 35K55, 35K60, 35K65, 35M10, 35Q80.

\section{InTRODUCTION}

In this paper we consider the non-linear system of mixed type

$$
\begin{gathered}
\frac{\partial u}{\partial t}+A\left(x, \frac{\partial}{\partial x}\right) u+\mathscr{R}(u)=f, \quad x \in \Omega \subset \mathbb{R}^{2}, t \in(0, T) \\
\left.\frac{\partial v}{\partial n}\right|_{x \in \partial \Omega}=0, \quad u(x, 0)=u_{0}(x),
\end{gathered}
$$

in a bounded, polygonal domain $\Omega \subset \mathbb{R}^{2}$ for the vector $u=(v, w)$; the data of the problem, $f(x, t)$ and $u_{0}(x)$, have a similar structure: $f=(g, h), u_{0}=\left(v_{0}, w_{0}\right)$. Here $A$ is a matrix differential operator of the form

$$
A=\left(\begin{array}{ll}
L\left(\frac{\partial}{\partial x}\right) & \ell_{1}(x) \\
\ell_{2}(x) & \ell_{3}(x)
\end{array}\right)
$$

where $\ell_{1}(x), \ell_{2}(x), \ell_{3}(x)$ are given functions, $L\left(\frac{\partial}{\partial x}\right)=-\Delta+I$ and $\mathscr{R}(u)=$ $(\mathscr{P}(u), \mathscr{Q}(u))$ is a vector field of non-linear terms. We assume that $\mathscr{P}$ is a linear combination of the terms $p_{j}, j=1, \ldots, 7$, satisfying conditions (1)-(7) in Section 7. These conditions are satisfied if $p_{i}$ are polynomials of degree $\geqslant 2$. The nonlinear operator 2 is a linear combination of terms $q_{j}$ given by 
(8) $q_{8}(v, w)=w^{m} v$,

(9) $q_{9}(w)=w^{m+1}$,

where $m$ is a positive integer.

Systems of this type have been used to model several phenomena in different fields, for instance in Physics in the studies of nuclear reactor dynamics and heat conduction (with adiabatic feedback effect in the reactor system) (see [32] chap. 1.3), in Neurophysiology in the formulation due to FritzHugh-Nagumo describing the ionic and electrical events occurring during the transmission of an impulse along an axon (see [32] chap. 12.7). Similar equations come from ecological applications, such as studies of forestry ecosystems (see [8], [9], [10], [25] and [38]), as well as from biological applications (see [29], chap. 13, [30], chap. 1, chap. 13 and [26]). A simpler version of problem (1.1) is introduced in [30], chap. 13, as a model of rabies epidemics. The population consists of two types of foxes: the vector $u$ is the couple $(v, w)$ where $v(x, t)$ is the density of the infective ones and $w(x, t)$ of the susceptible ones, the non-linear terms reflect the interactions between the two types of foxes. The model takes into account the life expectancy of infective foxes, a measure of transmission efficiency of the disease from infective ones to susceptible ones and the diffusion coefficient of infected foxes. Neumann boundary condition on the infected foxes are given (i.e. the migration of cubs seeking their own territory is excluded).

In the classical setting of smooth domains in $\mathbb{R}^{n}$ coercive estimates of solutions of general parabolic initial-boundary value problems have been proved by Agranovich and Vishik in the Sobolev-Slobodetskii norms $W_{2}^{2 b l, l}[2]$ and coercive Schauder type estimates have been established by Solonnikov [36] and extended by Belonosov [5] to weighted Hölder spaces. Stability results have been proved by Henry by an abstract approach [19] and for a large class of non-linear parabolic systems by Belonosov and Višnevskiǐ [6].

Recently, stability and instability of a stationary solution for non-linear systems of mixed type (as in (1.1)) has been studied by Mulone and Solonnikov and a linearization principle in Sobolev-Slobodetskii spaces with an exponential weight have been proved [28]. In the paper [17] we studied non-linear mixed type systems of $2 m$-equations in $n$ spatial dimensions and we proved existence, uniqueness results and coercive estimates in the Hölder spaces for the solution of the associated linear mixed-type problem (see Theorem 2.1 in [17]). Assuming suitable conditions on the spectrum of the operator $-A$ and on the eigenvalues of matrix $-\ell_{3}$ we established weighted estimates in the Hölder spaces (see Theorem 2.2 in [17]). These estimates are crucial for proving the stability result for the nonlinear problem (see Theorem 3.1 in [17]).

In the present paper we study problem (1.1) in a non convex polygonal domain $\Omega \subset \mathbb{R}^{2}$. For simplicity we assume that the boundary of $\Omega$ is smooth everywhere except a single angular point $x=0$ with the aperture of the angle $\theta>\pi$. The case of a fixed (finite) number of angular points can be easily reduced to the case of a singular point by using partition of the unity. In order to extend the results of the present paper to a larger class of irregular domains - for instance $(\varepsilon, \delta)$-domains with fractal boundary - it would be important to understand as 
our results depend on the increasing number of sides of the polygonal boundaries approximating the fractal curve. This question, interesting in our opinion, is beyond the aim of this paper and it will be faced in a forthcoming paper.

We describe briefly the contents of the present article. First we consider linear parabolic Neumann problems. The Dirichlet and Neumann problems in irregular domains have been faced in many papers and books. We mention [35], [23], [24], [31] and we refer to the bibliography quoted there. In this paper we state existence, uniqueness results and coercive estimates in the space $W_{2, \mu}^{2,1}\left(Q_{T}\right)$ for the solution (see Theorem 5.1). The presence of angles with aperture $\theta>\pi$ causes a loss of regularity for the solutions of the (linear) elliptic problems and the $H^{2}$-regularity fails even in the case of smooth data. The natural setting in our geometry are then weighted Sobolev spaces where the weight $|x|^{\mu}$ is the distance from a single angular point 0 . Moreover because of the Neumann condition on the boundary the weighted Sobolev spaces involved are the spaces $W_{2, \mu}^{2,1}\left(Q_{T}\right)$, $Q_{T}=\Omega \times(0, T)$ (see formula (2.1)) which differ from the spaces of the Kondrat'iev type $H_{\mu}^{2,1}\left(Q_{T}\right)$. It is clear that the $H$-spaces are continuously imbedded in the corresponding $W$-spaces. From the Hardy inequalities it follows that the norms $\|u\|_{W_{2, \mu}^{1}(\Omega)}$ and $\|u\|_{H_{\mu}^{1}(\Omega)}$ are equivalent as well as the norms $\|u\|_{W_{2, \mu}^{2}(\Omega)}$ and $\|u\|_{H_{\mu}^{2}(\Omega)}$ for functions vanishing for $x=0$ and these spaces coincide. We also mention related papers [13], [14], [15] and [16] where elliptic and parabolic problems with oblique derivative conditions on the sides of an infinite angle have been studied, and [11], [12], devoted to the problems with dynamic boundary condition on one of the sides.

A technical difficulty arising in the Neumann problem consists in the fact that in general the solutions do not vanish for $x=0$, which makes it necessary to establish the special trace results for the spaces $W_{2, \mu}^{2,1}\left(Q_{T}\right)$ (see Propositions 4.1, 4.2 and 4.3). This enables us to reduce our problem to a similar one with zero initial datum by applying the above-mentioned trace results. Then we convert it by means of the Laplace transform in a parameter-dependent problem as in the article of Agranovich and Vishik [2] and we establish existence, uniqueness results and coercive estimates in the space $W_{2, \mu}^{2}(\Omega)$ for the solution, principal tools being the Lax-Milgram theorem, estimates obtained in [39] and Kondrat'iev's results. We study then the linear system of mixed type associated to problem (1.1) and we prove existence, uniqueness results and coercive estimates in the space $W_{2, \mu}^{2,1}\left(Q_{T}\right) \times W_{2}^{s, 0}\left(Q_{T}\right)$ (see Theorem 6.1). The proof is based on the analysis of the Cauchy problem related to the second equation of system (1.1) and on the previously mentioned results for the parabolic Neumann problem related to the first equation of system (1.1). More precisely we represent the solution of the Cauchy problem by means of the resolving operator, we plug the expression in the first equation and we obtain an initial-boundary value problem with an integral operator of Volterra type that we solve by successive approximations and Gronwall Lemma. Theorem 6.1 allows us to define the resolvent operator $e^{-A t}$. Assuming suitable conditions on the spectrum of the operator $-A$ and on the coefficient $\ell_{3}$ we establish the exponential decay of the operator $e^{-A t}$ (Theorem 6.2). The proof of Theorem 6.2 is in some sense the most delicate part of this paper, 
principal tools being sharp estimates for the parameter-dependent problem (Theorem 5.2), fixed point arguments, the Gohberg theorem (Theorem 1.5.1 in [18]), Fredholm alternative theorem, compact imbedding results in the weighted spaces (Proposition 3.1), and the results of Henry (Theorem 1.3.4 in [19]). Theorems 6.1 and 6.2 allow us to prove the exponential decay of the solution $u$ of a linear problem in the spaces $W_{2, \mu}^{2,1}\left(Q_{T}\right) \times W_{2}^{s, 0}\left(Q_{T}\right)$ (Theorem 6.3). These estimates, that to our mind are interesting in themselves, are crucial for establishing the stability result for the non-linear problem (Theorem 7.1).

The layout of this paper is the following: in Section 2 we introduce the spaces involved in our setting, in Section 3 we prove inclusions results, in Section 4 trace results. Section 5 concerns Neumann parabolic problems. In Section 6 we deal with the linear mixed-type system related to problem (1.1). Finally Section 7 concerns problem (1.1).

\section{NotATiOn}

In this section we introduce the weighted Sobolev spaces involved in our results. Let $\Omega \subset \mathbb{R}^{2}$ denote a bounded domain whose boundary is smooth everywhere except a single angular point $x=0$. We suppose that the aperture of the angle, denoted by $\theta$, is strictly greater than $\pi$. For simplicity we assume that in a certain neighborhood of the angular point the boundary of $\Omega$ is formed by two straight segments.

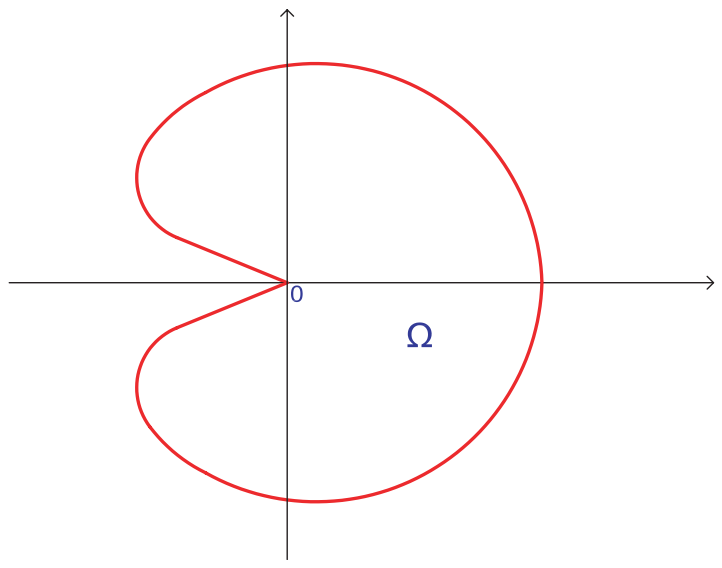

Set $Q_{T}=\Omega \times(0, T)$ and $\mu \in(0,1)$. Let $L_{q, \mu}\left(Q_{T}\right)$ denote the completion of the space $C^{0}\left(\bar{Q}_{T}\right)$ with respect to the norm

$$
\|v\|_{L_{q, \mu}\left(Q_{T}\right)}=\left\{\int_{0}^{T} \int_{\Omega}|v(x, t)|^{q}|x|^{q \mu} d x d t\right\}^{1 / q},
$$


then the space

$$
W_{2, \mu}^{2,1}\left(Q_{T}\right)=\left\{v \in L_{2, \mu}\left(Q_{T}\right): D_{x}^{\alpha} D_{t}^{k} v \in L_{2, \mu}\left(Q_{T}\right), \forall|\alpha|+2 k \leqslant 2\right\}
$$

is a Hilbert space with the norm

$$
\|v\|_{W_{2, \mu}^{2,1}\left(Q_{T}\right)}=\left\{\sum_{|\alpha|+2 k \leqslant 2}\left\|D_{x}^{\alpha} D_{t}^{k} v\right\|_{L_{2, \mu}\left(Q_{T}\right)}^{2}\right\}^{1 / 2}
$$

$\alpha=\left(\alpha_{1}, \alpha_{2}\right), \alpha_{1}, \alpha_{2}, k \in \mathbb{N} \cup 0$.

The space

$$
W_{2}^{s, 0}\left(Q_{T}\right)=L_{2}\left(0, T ; W_{2}^{s}(\Omega)\right)
$$

is a Hilbert space with the norm

$$
\|v\|_{W_{2}^{s, 0}\left(Q_{T}\right)}=\left\{\int_{0}^{T}\|v\|_{W_{2}^{s}(\Omega)}^{2} d t\right\}^{1 / 2}
$$

where $W_{2}^{s}(\Omega)$ denotes the usual (possibly fractional) Sobolev space on $\Omega, s \geqslant 0$. If $s=[s]+\sigma, 0<\sigma<1$, then

$$
\|v\|_{W_{2}^{s}(\Omega)}^{2}=\sum_{|\alpha| \leq[s]}\left\|D^{\alpha} v\right\|_{L_{2}(\Omega)}^{2}+\sum_{|\alpha|=[s]} \int_{\Omega} \int_{\Omega} \frac{\left|D^{\alpha} v(x)-D^{\alpha} v(y)\right|^{2}}{|x-y|^{2+2 \sigma}} d x d y
$$

(see e.g. [1]). Finally, $W_{2, \mu}^{k}(\Omega)$ denotes the weighted Sobolev space that is a Hilbert with the norm

$$
\|v\|_{W_{2, \mu}^{k}(\Omega)}=\left\{\sum_{|\alpha| \leqslant k}\left\|D^{\alpha} v\right\|_{L_{2, \mu}(\Omega)}^{2}\right\}^{1 / 2} .
$$

\section{INCLUSION RESULTS}

We state now some inclusion results that are important tools in our estimates. From now on, we denote by $c$ (possibly) different constants.

Let $\mathscr{D}$ be an infinite wedge with the vertex at the origin and aperture $\theta>\pi$ (we may assume without loss of generality that $\mathscr{D}$ is symmetric with respect to the $x_{1}$-axis and it contains the half-axis $\left.x_{1}>0\right)$. By $\mathbb{C}_{x}$ we mean an infinite wedge with the vertex $x$ and aperture $\theta_{1}=2 \pi-\theta$ oriented in the same way as $\mathscr{D}$, and $\mathbb{C}_{x}\left(d_{0}\right)=\left\{y \in \mathbb{C}_{x},|y-x| \leq d_{0}\right\}$. If $x \in \mathscr{D}$, then $\mathbb{C}_{x} \subset \mathscr{D}$.

For arbitrary differentiable function $u(x)$ vanishing at infinity the Smith representation formula holds (see [34])

$$
u(x)=\int_{\mathbb{C}_{x}} \mathbf{K}(x-y) \cdot \nabla u(y) d y,
$$


where

$$
\mathbf{K}(z)=\frac{z}{|z|^{2}} \omega\left(\frac{z}{|z|}\right) .
$$

The function $\omega(\xi)$ defined on the unit sphere $S_{1}$ is smooth and its support is contained in $\mathbb{C}_{0} \cap S_{1}$, in addition, $\int_{S_{1}} \omega(\xi) d S=1$. Hence $\mathbf{K}$ vanishes on the surface of $\mathbb{C}_{0}$. It can be extended by zero in $\mathbb{R}^{2} \backslash \mathbb{C}_{0}$; the extended function is smooth everywhere except the vertex and

$$
\left|D^{\alpha} \mathbf{K}(z)\right| \leq \frac{c}{|z|^{1+|\alpha|}}
$$

where $\alpha=\left(\alpha_{1}, \alpha_{2}\right), \alpha_{i} \in \mathbb{N} \cup 0$.

The domain $\Omega$ described in Section 2 possesses the cone property which means that with every point $x \in \Omega$ we can associate a finite cone $\mathbb{C}_{x}\left(d_{0}\right) \subset \Omega$ with a fixed (independent of $x$ ) aperture $\theta_{1}$ and fixed $d_{0}>0$ (it may be oriented in an arbitrary way). From the Smith representation formula mentioned above it follows that arbitrary $v(x) \in W_{2, \mu}^{1}(\Omega)$ can be represented in the form

$$
\begin{aligned}
v(x) & =\int_{\mathbb{C}_{x}\left(d_{0}\right)} \mathbf{K}_{1}(x-y) \nabla v(y) d y+\int_{\mathbb{C}_{x}\left(d_{0}\right)} K_{2}(x-y) v(y) d y \\
& \equiv v_{1}(x)+v_{2}(x),
\end{aligned}
$$

where

$$
\begin{aligned}
& \mathbf{K}_{1}(x-y)=\mathbf{K}(x-y) \psi\left(|x-y| / d_{0}\right) \\
& K_{2}(x-y)=-\operatorname{div}\left(\mathbf{K}(x-y)\left(1-\psi\left(|x-y| / d_{0}\right)\right)\right)
\end{aligned}
$$

and $\psi(r)$ is a monotone function of a positive argument $r>0$ equal to 1 for $r<1 / 2$ and to 0 for $r>3 / 4$. By (3.2),

$$
\left|\mathbf{K}_{1}(x-y)\right| \leq c|x-y|^{-1}, \quad\left|K_{2}(x-y)\right| \leq c d_{0}^{-2}
$$

and $\operatorname{supp}_{y} \mathbf{K}_{1}(x-y) \subset \mathbb{C}_{x}\left(d_{0}\right), \operatorname{supp}_{y} K_{2}(x-y) \subset \mathbb{C}_{x}\left(d_{0}\right) \backslash \mathbb{C}_{x}\left(d_{0} / 2\right)$.

PROPOSITION 3.1. If $2 \leq q<\frac{2(1+\mu)}{\mu}$ then the following compact imbedding holds

$$
W_{2, \mu}^{1}(\Omega) \subset L_{q, 2 \mu / q}(\Omega),
$$

with

$$
\|v\|_{L_{q, 2 \mu / q}(\Omega)} \leqslant c\|v\|_{W_{2, \mu}^{1}(\Omega)} .
$$

If $2 \leq q<\frac{2 \sigma}{(\sigma-1) \mu}, \sigma \geq 1$, then

$$
W_{2, \mu}^{1}(\Omega) \subset L_{q, \mu / \sigma}(\Omega),
$$


with

$$
\|v\|_{L_{q, \mu / \sigma}(\Omega)} \leqslant c\|v\|_{W_{2, \mu}^{1}(\Omega)} .
$$

ProOF. We consider the case $q>2$. We represent $v(x)$ in the form (3.3) and estimate $v_{1}(x)$. By the Hölder inequality,

$$
\begin{aligned}
\left|v_{1}(x)\right| \leq & \left(\int_{\mathbb{C}_{x}\left(d_{0}\right)}\left|\mathbf{K}_{1}(x-y)\right|^{a}|\nabla v|^{2}|y|^{2 \mu} d y\right)^{1 / q} \\
& \cdot\left(\int_{\mathbb{C}_{x}\left(d_{0}\right)}\left|\mathbf{K}_{1}(x-y)\right|^{b}|y|^{-2 \mu} d y\right)^{1 / 2} \\
& \cdot\left(\int_{\mathbb{C}_{x}\left(d_{0}\right)}|\nabla v|^{2}|y|^{2 \mu} d y\right)^{1 / 2-1 / q},
\end{aligned}
$$

where $0<a<2,0<b<2, \frac{a}{q}+\frac{b}{2}=1, q>2$. Actually we choose

$$
b=2(1-\varepsilon), \quad a=q \varepsilon,
$$

where $\varepsilon$ denotes a positive, sufficiently small constant. By (3.5),

$$
\int_{\mathbb{C}_{x}\left(d_{0}\right)}\left|\mathbf{K}_{1}(x-y)\right|^{b}|y|^{-2 \mu} d y \leq c \int_{\mathbb{R}^{2}} \frac{d y}{|x-y|^{b}|y|^{2 \mu}} \leq c|x|^{-b-2 \mu+2},
$$

and we evaluate the norm of $v_{1}$ coming back to the inequality (3.8). We have

$$
\begin{aligned}
& \int_{\Omega}\left|v_{1}(x, t)\right|^{q}|x|^{2 \mu} d x \leq c\left(\int_{\Omega}|\nabla v|^{2}|y|^{2 \mu} d y\right)^{q / 2-1} \\
& \quad \int_{\Omega}|x|^{-(b+2 \mu-2) q / 2+2 \mu} d x \int_{\mathbb{C}_{x}\left(d_{0}\right)}\left|\mathbf{K}_{1}(x-y)\right|^{a}|\nabla v|^{2}|y|^{2 \mu} d y \\
& \leq c\|\nabla v\|_{L_{2, \mu}(\Omega)}^{q-2} \int_{\Omega}|\nabla v(y)|^{2}|y|^{2 \mu} d y \int_{|y-x| \leq d_{0}} \frac{|x|^{2 \mu} d x}{|x-y|^{a}|x|^{(b+2 \mu-2) q / 2}} \\
& \leq c d_{0}^{2(1+\mu)-q \mu}\|\nabla v\|_{L_{2, \mu}(\Omega)}^{q},
\end{aligned}
$$

because $(-b-2 \mu+2) q / 2+2 \mu=q(\varepsilon-\mu)+2 \mu \in(-2,0)$ and

$$
(-b-2 \mu+2) q / 2+2 \mu+2-a=-q \varepsilon+2-q+q \varepsilon-q \mu+q+2 \mu>0
$$

for $q<\frac{2(1+\mu)}{\mu}$; hence

$$
\int_{|x-y| \leq d_{0}}|x-y|^{-a}|x|^{(-b-2 \mu+2) q / 2+2 \mu} d x \leq c d_{0}^{2+2 \mu-q \mu}
$$

for arbitrary $y \in \Omega$. 
Hence

$$
\left\|v_{1}\right\|_{L_{q, 2 \mu / q}(\Omega)} \leq c d_{0}^{2(1+\mu) / q-\mu}\|\nabla v\|_{L_{2, \mu}(\Omega)} .
$$

By exactly the same arguments we show that

$$
\left\|v_{2}\right\|_{L_{q, 2 \mu / q}(\Omega)} \leq c d_{0}^{2(1+\mu) / q-\mu-1}\|v\|_{L_{2, \mu}(\Omega)},
$$

and (3.6) is proved.

Replacing $d_{0}$ with $\epsilon d_{0}, \epsilon \ll 1$, we obtain

$$
\|v\|_{L_{q, 2 \mu / q}(\Omega)} \leq c\left(\epsilon^{2(1+\mu) / q-\mu}\|\nabla v\|_{L_{2, \mu}(\Omega)}+\epsilon^{2(1+\mu) / q-\mu-1}\|v\|_{L_{2, \mu}(\Omega)}\right) .
$$
at

To prove (3.7), we repeat the above calculations and arrive, instead of (3.10),

$$
\begin{aligned}
& \int_{\Omega}\left|v_{1}(x, t)\right|^{q}|x|^{q \mu / \sigma} d x \leq c \mid\|\nabla v\|_{L_{2, \mu}(\Omega)} \\
& \quad \cdot \int_{\Omega}|\nabla v|^{2}|y|^{2 \mu} d y \int_{|y-x| \leq d_{0}}|x-y|^{-a}|x|^{(-b-2 \mu+2) q / 2+q \mu / \sigma} d x \\
& \leq c d_{0}^{q \gamma}\|\nabla v\|_{L_{2, \mu}(\Omega)}^{q},
\end{aligned}
$$

where $\gamma=q \mu \frac{1-\sigma}{\sigma}+2>0$, if $\sigma>1$, and $\gamma=2$, if $\sigma=1$.

We also have

$$
\int_{\Omega}\left|v_{2}(x, t)\right|^{q}|x|^{q \mu / \sigma} d x \leq c d_{0}^{q(\gamma-1)}\|v\|_{L_{2, \mu}(\Omega)}^{q}
$$

which completes the proof of (3.7).

Along with (3.7), there holds

$$
\|v\|_{L_{q, \mu / \sigma}(\Omega)} \leq c\left(\epsilon^{\gamma}\|\nabla v\|_{L_{2, \mu}(\Omega)}+\epsilon^{\gamma-1}\|v\|_{L_{2, \mu}(\Omega)}\right), \quad \epsilon \ll 1 .
$$

The proposition is proved.

By similar arguments we can prove the inequality

$$
\|\nabla v\|_{L_{2, \mu}(\Omega)} \leq c\left(d_{0}\left\|D^{2} v\right\|_{L_{2, \mu}(\Omega)}+d_{0}^{-1}\|v\|_{L_{2, \mu}(\Omega)}\right),
$$

where $D^{2} v=\left(\frac{\partial^{2} v}{\partial x_{i} \partial x_{j}}\right)_{i, j=1,2}$. Instead of (3.3), we should use the representation formula

$$
\frac{\partial v(x)}{\partial x_{i}}=\int_{\mathbb{C}_{x}\left(d_{0}\right)} \mathbf{K}_{1}(x-y) \nabla\left(\frac{\partial v(y)}{\partial y_{i}}\right) d y+\int_{\mathbb{C}_{x}\left(d_{0}\right)} K_{3}^{i}(x-y) v(y) d y
$$

where $K_{3}^{i}(x-y)=\frac{\partial K_{2}(x-y)}{\partial x_{i}}$ and $K_{2}(x-y)$ is defined in (3.4). This kernel satisfies the inequality

$$
\left|K_{3}^{i}(x-y)\right| \leq c d_{0}^{-3}
$$


REMARK 3.1. We note that inequality (3.13) still holds for functions $v$ defined in the infinite wedge $\mathscr{D}$ if we replace the domain $\Omega$ by the wedge $\mathscr{D}$ and the constant $d_{0}$ by any positive number $\xi$.

Proposition 3.2. If $0 \leq s<2-\mu$, then the following imbedding holds

$$
W_{2, \mu}^{2}(\Omega) \subset W_{2}^{s}(\Omega)
$$

with

$$
\|v\|_{W_{2}^{s}(\Omega)} \leqslant c\|v\|_{W_{2, \mu}^{2}(\Omega)}
$$

and this implies that

$$
\|v\|_{W_{2}^{s, 0}\left(Q_{T}\right)}^{2} \leqslant c \int_{0}^{T}\|v(\cdot, t)\|_{W_{2, \mu}^{2}(\Omega)}^{2} d t=c\|v\|_{W_{2, \mu}^{2,0}\left(Q_{T}\right)}^{2} .
$$

Moreover, if $s>1$ then

$$
\sup _{x}|v|^{2} \leqslant c\|v\|_{W_{2}^{s}(\Omega)}^{2}
$$

Proof. We start by showing that

$$
\|u\|_{W_{2}^{\sigma}(\Omega)} \leq c\|u\|_{W_{2, \mu}^{1}(\Omega)}, \quad \sigma \in[0,1-\mu) .
$$

The inequality

$$
\|u\|_{L_{2}(\Omega)} \leq c\left(d_{0}^{1-\mu}\|\nabla u\|_{L_{2, \mu}(\Omega)}+d_{0}^{-\mu}\|u\|_{L_{2, \mu}(\Omega)}\right)
$$

is obtained in the same way as (3.6), (3.7). Now we represent $u$ as the sum $u(x)=u(x) \psi\left(x / d_{1}\right)+u(x)\left(1-\psi\left(x / d_{1}\right)\right):=u_{1}+u_{2}$ where $\psi$ is a smooth monotone function of $|x|$ equal to 1 for $|x| \leq 3 / 4$ and to zero for $|x| \geq 1$. The constant $d_{1}$ is chosen in such a way that the boundary of $\Omega$ consists of two straight lines for $|x| \leq d_{1}$, and $d_{1}>d_{0}$. We can consider $u_{1}(x)=u(x) \psi\left(x / d_{1}\right)$ as a function given in $\mathscr{D}$, setting $u_{1}(x)=0$ for $|x|>d_{1}$. Let us estimate the seminorm

$$
\left\|u_{1}\right\|_{\dot{W}_{2}^{\sigma}(\mathscr{D})}=\left(\int_{\mathscr{D}} \int_{\mathscr{D}} \frac{\left|u_{1}(x)-u_{1}(z)\right|^{2}}{|x-z|^{2+2 \sigma}} d x d z\right)^{1 / 2}
$$

It is easily seen that

$$
\left(\int_{\mathscr{D}} \int_{\mathscr{D},|x-z|>d_{0}} \frac{\left|u_{1}(x)-u_{1}(z)\right|^{2}}{|x-z|^{2+2 \sigma}} d x d z\right)^{1 / 2} \leq c d_{0}^{-\sigma}\left\|u_{1}\right\|_{L_{2}(\Omega)}
$$

and

$$
d_{0}^{-\sigma}\left\|u_{1}\right\|_{L_{2}(\Omega)} \leq c\left(d_{0}^{1-\mu-\sigma}\left\|\nabla u_{1}\right\|_{L_{2, \mu}(\Omega)}+d_{0}^{-\mu-\sigma}\left\|u_{1}\right\|_{L_{2, \mu}(\Omega)}\right) .
$$

Now we estimate the integral

$$
\int_{\mathscr{D}} \int_{\mathscr{D},|x-z|<d_{0}} \frac{\left|u_{1}(x)-u_{1}(z)\right|^{2}}{|x-z|^{2+2 \sigma}} d x d z
$$


By (3.1),

$$
\begin{aligned}
u_{1}(x)-u_{1}(z)= & \int_{\mathbb{C}_{x}} \mathbf{K}(x-y) \cdot \nabla u_{1}(y) d y-\int_{\mathbb{C}_{z}} \mathbf{K}(z-y) \cdot \nabla u_{1}(y) d y \\
= & \int_{\mathbb{C}_{x} \cap B_{4 r}(X)} \mathbf{K}(x-y) \cdot \nabla u_{1}(y) d y-\int_{\mathbb{C}_{z} \cap B_{4 r}(X)} \mathbf{K}(z-y) \cdot \nabla u_{1}(y) d y \\
& +\int_{\left(\mathbb{C}_{x} \cup \mathbb{C}_{z}\right) \backslash B_{4 r}(X)}(\mathbf{K}(x-y)-\mathbf{K}(z-y)) \cdot \nabla u_{1}(y) d y \\
:= & I_{1}+I_{2}+I_{3},
\end{aligned}
$$

where $r=|x-z|, X=(x+z) / 2, B_{a}(X)$ is a circle of radius $a$ centered at $X$. In the integral $I_{3}$, the kernels $\mathbf{K}(x-y)$ and $\mathbf{K}(z-y)$ are extended by zero in $\mathbb{R}^{2}$ (with respect to the variable $y$ ).

It is easily verified that

$$
\mathbb{C}_{x} \cap B_{4 r}(X) \subset \mathbb{C}_{x}(9 r / 2), \quad \mathbb{C}_{z} \cap B_{4 r}(X) \subset \mathbb{C}_{z}(9 r / 2),
$$

hence

$$
\begin{aligned}
\left|I_{1}\right|^{2} & \leq c \int_{\mathbb{C}_{x}(9 r / 2)}|\mathbf{K}(x-y)|^{1+\kappa}\left|\nabla u_{1}(y)\right|^{2}|y|^{2 \mu} d y \int_{\mathbb{C}_{x}(9 r / 2)}|\mathbf{K}(x-y)|^{1-\kappa}|y|^{-2 \mu} d y \\
& \leq c r^{1+\kappa-2 \mu} \int_{\mathbb{C}_{x}(9 r / 2)}|\mathbf{K}(x-y)|^{1+\kappa}\left|\nabla u_{1}(y)\right|^{2}|y|^{2 \mu} d y,
\end{aligned}
$$

where $\max (0,2 \mu-1)<\kappa<\mu$, and

$$
\begin{aligned}
& \int_{\mathscr{D}} \int_{\mathscr{D},|x-z|<d_{0}}\left|I_{1}\right|^{2} \frac{d x d z}{|x-z|^{2+2 \sigma}} \\
& \quad \leq\left. c \int_{\mathscr{D}} \int_{\mathscr{D},|x-z|<d_{0}} \frac{d x d z}{|x-z|^{1+2 \sigma+2 \mu-\kappa}} \int_{\mathbb{C}_{x}(9 r / 2)}|\mathbf{K}(x-y)|^{1+\kappa}\left|\nabla u_{1}(y)\right|^{2}|| y\right|^{2 \mu} d y .
\end{aligned}
$$

We introduce in the last integral a new variable of integration $\xi=z-x$ instead of $z$ and obtain

$$
\begin{aligned}
& \int_{\mathscr{D}} \int_{\mathscr{D},|x-z|<d_{0}}\left|I_{1}\right|^{2} \frac{d x d z}{|x-z|^{2+2 \sigma}} \\
& \quad \leq c \int_{\mathscr{D}}\left|\nabla u_{1}\right|^{2}|y|^{2 \mu} d y \int_{|\xi| \leq d_{0}} \frac{d \xi}{|\xi|^{1+2 \sigma+2 \mu-\kappa}} \int_{|x-y| \leq(9 / 2)|\xi|}|x-y|^{-1-\kappa} d x \\
& \quad \leq c d_{0}^{2-2 \mu-2 \sigma} \int_{\mathscr{D}}\left|\nabla u_{1}\right|^{2}|y|^{2 \mu} d y
\end{aligned}
$$


In the same way we show that

$$
\int_{\mathscr{D}} \int_{\mathscr{D},|x-z|<d_{0}}\left|I_{2}\right|^{2} \frac{d x d z}{|x-z|^{2+2 \sigma}} \leq c d_{0}^{2-2 \mu-2 \sigma} \int_{\mathscr{D}}\left|\nabla u_{1}\right|^{2}|y|^{2 \mu} d y .
$$

Now we pass to the estimate of $I_{3}$. If $y \in\left(\mathbb{C}_{x} \cup \mathbb{C}_{z}\right) \backslash B_{4 r}(X)$, then

$$
|\mathbf{K}(x-y)-\mathbf{K}(z-y)| \leq c|x-z||X-y|^{-2},
$$

hence

$$
\begin{aligned}
\left|I_{3}\right| \leq & c|x-z| \int_{\left(\mathbb{C}_{x} \cup \mathbb{C}_{z}\right) \backslash B_{4 r}(X)}|X-y|^{-2}\left|\nabla u_{1}(y)\right| d y \\
\leq & c|x-z|\left(\int_{\mathscr{D} \backslash B_{4|x-z|}(X)}\left|\nabla u_{1}(y)\right|^{2} \frac{|y|^{2 \mu} d y}{|X-y|^{2+2 v}}\right)^{1 / 2} \\
& \times\left(\int_{|X-y| \geq 4|x-z| \mid} \frac{\left.d y\right|^{2 \mu}|X-y|^{2-2 v}}{\mid 1 / 2}\right. \\
\leq & c|x-z|^{1+v-\mu}\left(\int_{\mathscr{D} \backslash B_{4|x-z|}(X)}\left|\nabla u_{1}(y)\right|^{2} \frac{|y|^{2 \mu} d y}{|X-y|^{2+2 v}}\right)^{1 / 2}
\end{aligned}
$$

where $0<v<\mu$, and

$$
\begin{aligned}
& \int_{\mathscr{D}} \int_{\mathscr{D},|x-z|<d_{0}}\left|I_{3}\right|^{2} \frac{d x d z}{|x-z|^{2+2 \sigma}} \\
& \quad \leq c \int_{\mathscr{D}} \int_{\mathscr{D},|x-z|<d_{0}} \frac{d x d z}{|x-z|^{2 \sigma+2 \mu-2 v}} \int_{\mathscr{D} \backslash B_{4|x-z|}(X)}\left|\nabla u_{1}(y)\right|^{2} \frac{|y|^{2 \mu} d y}{|X-y|^{2+2 v}} .
\end{aligned}
$$

We introduce new variables of integration

$$
X=(x+z) / 2, \quad \xi=(x-z) / 2
$$

and obtain

$$
\begin{aligned}
& \int_{\mathscr{D}} \int_{\mathscr{D},|x-z|<d_{0}}\left|I_{3}\right|^{2} \frac{d x d z}{|x-z|^{2+2 \sigma}} \\
& \quad \leq c \int_{|\xi| \leq d_{0}} \frac{d \xi}{|\xi|^{2(\sigma+\mu-v)}} \int_{\mathscr{D}}\left|\nabla u_{1}\right|^{2}|y|^{2 \mu} d y \int_{|X-y| \geq 4|\xi|} \frac{d X}{|X-y|^{2+2 v}} \\
& \quad \leq c d_{0}^{2(1-\sigma-\mu)}\left\|\nabla u_{1}\right\|_{L_{2, \mu}(\mathscr{D})}^{2} .
\end{aligned}
$$

Hence by (3.20), (3.21), (3.22), (3.18) and (3.19)

$$
\left\|u_{1}\right\|_{\dot{W}_{2}^{\sigma}(\mathscr{D})} \leq c\left(d_{0}^{1-\sigma-\mu}\left\|\nabla u_{1}\right\|_{L_{2, \mu}(\mathscr{D})}+d_{0}^{-\sigma-\mu}\left\|u_{1}\right\|_{L_{2, \mu}(\mathscr{D})}\right) .
$$


Now we pass to the estimate of $u_{2}(x)=u(x)\left(1-\psi\left(x / d_{1}\right)\right)$. Since $u_{2}(x)=0$ for $|x| \leq 3 d_{1} / 4$, it suffices to estimate this function in $\Omega^{\prime}=\left\{x \in \Omega:|x| \geq d_{1} / 2\right\}$. It is known that (see e.g. [27])

$$
\left\|u_{2}\right\|_{\dot{W}_{2}^{\sigma}\left(\Omega^{\prime}\right)} \leq c\left(d_{0}^{1-\sigma}\left\|\nabla u_{2}\right\|_{L_{2}\left(\Omega^{\prime}\right)}+d_{0}^{-\sigma}\left\|u_{2}\right\|_{L_{2}\left(\Omega^{\prime}\right)}\right),
$$

and since $|x| \geq d_{0} / 2$ in $\Omega^{\prime}$, we also have

$$
\left\|u_{2}\right\|_{\dot{W}_{2}^{\sigma}\left(\Omega^{\prime}\right)} \leq c\left(d_{0}^{1-\sigma-\mu}\left\|\nabla u_{2}\right\|_{L_{2, \mu}\left(\Omega^{\prime}\right)}+d_{0}^{-\sigma-\mu}\left\|u_{2}\right\|_{L_{2, \mu}\left(\Omega^{\prime}\right)}\right) .
$$

Together with (3.23), (3.17), this inequality yields

$$
\|u\|_{W_{2}^{\sigma}(\Omega)} \leq c\left(d_{0}^{1-\sigma-\mu}\|\nabla u\|_{L_{2, \mu}(\Omega)}+d_{0}^{-\sigma-\mu}\|u\|_{L_{2, \mu}(\Omega)}\right),
$$

hence (3.16) is proved. In addition, replacing $d_{0}$ with $\epsilon d_{0}$, we obtain

$$
\|u\|_{W_{2}^{\sigma}(\Omega)} \leq c\left(\epsilon^{1-\sigma-\mu}\|\nabla u\|_{L_{2, \mu}(\Omega)}+\epsilon^{-\sigma-\mu}\|u\|_{L_{2, \mu}(\Omega)}\right) .
$$

Inequality (3.14) is a consequence of (3.24), (3.13). Indeed, taking $\sigma=s-1$, we obtain

$$
\begin{aligned}
\|\nabla v\|_{W_{2}^{s-1}(\Omega)} & \leq c\left(d_{0}^{2-s-\mu}\left\|D^{2} v\right\|_{L_{2, \mu}(\Omega)}+d_{0}^{1-s-\mu}\|\nabla v\|_{L_{2, \mu}(\Omega)}\right) \\
& \leq c\left(d_{0}^{2-s-\mu}\left\|D^{2} v\right\|_{L_{2, \mu}(\Omega)}+d_{0}^{-s-\mu}\|v\|_{L_{2, \mu}(\Omega)}\right) .
\end{aligned}
$$

We also have

$$
\|v\|_{W_{2}^{s}(\Omega)} \leq \epsilon\|v\|_{W_{2, \mu}^{2}(\Omega)}+c(\epsilon)\|v\|_{L_{2, \mu}(\Omega)}, \quad \epsilon \ll 1 .
$$

Finally, (3.15) follows from (3.14) and from the imbedding of $W_{2}^{s}(\Omega), s>1$, in $C^{0}(\bar{\Omega})$. The proposition is proved.

REMARK 3.2. We note that for $s>1$ the Sobolev space $W_{2}^{s}(\Omega)$ is an algebra with respect to the product of functions, because of the Sobolev-Slobodeckil embedding results. Moreover it holds for $1<s<2-\mu$

$$
\begin{array}{rl}
\|u v\|_{W_{2}^{s}(\Omega)} \leqslant c & c\|u\|_{W_{2}^{s}(\Omega)}\left(\|v\|_{W_{2}^{1}(\Omega)}+\|v\|_{L_{\infty}(\Omega)}\right) \\
& \left.+\|v\|_{W_{2}^{s}(\Omega)}\left(\|u\|_{W_{2}^{1}(\Omega)}+\|u\|_{L_{\infty}(\Omega)}\right)\right)
\end{array}
$$

Indeed,

$$
\begin{gathered}
\|u v\|_{L_{2}(\Omega)} \leqslant c\|u\|_{L_{\infty}(\Omega)}\|u\|_{L_{2}(\Omega)} \\
\|\nabla(u v)\|_{L_{2}(\Omega)} \leqslant c\left(\|u\|_{L_{\infty}(\Omega)}\|v\|_{W_{2}^{1}(\Omega)}+\|v\|_{L_{\infty}(\Omega)}\|u\|_{W_{2}^{1}(\Omega)}\right) \\
\int_{\Omega} \int_{\Omega}|u(x)|^{2}|\nabla v(x)-\nabla v(y)|^{2} \frac{d x d y}{|x-y|^{2 s}} \leq c\|u\|_{L_{\infty}(\Omega)}^{2}\|v\|_{W_{2}^{s}(\Omega)}^{2}
\end{gathered}
$$


Let us show that

$$
\int_{\Omega} \int_{\Omega}|\nabla v(x)|^{2}|u(x)-u(y)|^{2} \frac{d x d y}{|x-y|^{2 s}} \leq c\|v\|_{W_{2}^{s}(\Omega)}^{2}\|u\|_{W_{2}^{1}(\Omega)}^{2} .
$$

Denoting by $u^{*}$ and $v^{*}$ the extensions of the functions $u$ and $v$ to $W_{2}^{s}\left(\mathbb{R}^{2}\right)$ such that

$$
\left\|u^{*}\right\|_{W_{2}^{s}\left(\mathbb{R}^{2}\right)} \leq c\|u\|_{W_{2}^{s}(\Omega)}, \quad\left\|u^{*}\right\|_{W_{2}^{1}\left(\mathbb{R}^{2}\right)} \leq c\|u\|_{W_{2}^{1}(\Omega)}
$$

and

$$
\left\|v^{*}\right\|_{W_{2}^{s}\left(\mathbb{R}^{2}\right)} \leq c\|v\|_{W_{2}^{s}(\Omega)}, \quad\left\|v^{*}\right\|_{W_{2}^{1}\left(\mathbb{R}^{2}\right)} \leq c\|v\|_{W_{2}^{1}(\Omega)}
$$

we obtain

$$
\begin{aligned}
& \int_{\Omega} \int_{\Omega}|\nabla v(x)|^{2}|u(x)-u(y)|^{2} \frac{d x d y}{|x-y|^{2 s}} \\
& \quad \leq \int_{\mathbb{R}^{2}} \frac{d z}{|z|^{2 s}} \int_{\mathbb{R}^{2}}\left|\nabla v^{*}(x)\right|^{2}\left|u^{*}(x+z)-u^{*}(x)\right|^{2} d x \\
& \quad \leq c\left\|\nabla v^{*}\right\|_{W_{2}^{s}\left(\mathbb{R}^{2}\right)}^{2} \int_{\mathbb{R}^{2}}\left\|u^{*}(\cdot+z)-u^{*}(\cdot)\right\|_{W_{2}^{2-s}\left(\mathbb{R}^{2}\right)}^{2} \frac{d z}{|z|^{2 s}}
\end{aligned}
$$

The last integral in (3.27) can be bounded by

$$
\begin{gathered}
c \int_{\mathbb{R}^{2}} \frac{d z}{|z|^{2 s}} \int_{\mathbb{R}^{2}}\left|e^{-i \xi \cdot z}-1\right|^{2}\left(1+|\xi|^{2}\right)^{2-s}\left|\hat{u}^{*}(\xi)\right|^{2} d \xi \\
\leq c \int_{\mathbb{R}^{2}}\left(1+\left.\xi\right|^{2}\right)\left|\hat{u}^{*}(\xi)\right|^{2} d \xi \leq c\left\|u^{*}\right\|_{W_{2}^{1}\left(\mathbb{R}^{2}\right)}^{2}
\end{gathered}
$$

where $\hat{u}^{*}$ is the Fourier transform of $u^{*}$. Hence

$$
\int_{\Omega} \int_{\Omega}|\nabla v(x)|^{2}|u(x)-u(y)|^{2} \frac{d x d y}{|x-y|^{2 s}} \leq c\|v\|_{W_{2}^{s}(\Omega)}^{2}\|u\|_{W_{2}^{1}(\Omega)}^{2} .
$$

In the same way we obtain

$$
\int_{\Omega} \int_{\Omega}|\nabla u(x)|^{2}|v(x)-v(y)|^{2} \frac{d x d y}{|x-y|^{2 s}} \leq c\|u\|_{W_{2}^{s}(\Omega)}^{2}\|v\|_{W_{2}^{1}(\Omega)}^{2} .
$$

Collecting the previous estimate we obtain inequality (3.26).

\section{TRACE RESUlts}

In this section we prove some trace theorems for the space $W_{2, \mu}^{2,1}\left(Q_{T}\right)$. 
Proposition 4.1. (i) If $u \in W_{2, \mu}^{2,1}\left(Q_{T}\right)$, then $u(0, \cdot) \in W_{2}^{(1-\mu) / 2}(0, T)$ and the following estimate holds

$$
\|u(0, \cdot)\|_{W_{2}^{(1-\mu) / 2}(0, T)} \leqslant c\|u\|_{W_{2, \mu}^{2,1}\left(Q_{T}\right)} .
$$

(ii) For any function $\varphi \in W_{2}^{(1-\mu) / 2}(0, T)$ there exists a function $\Phi \in W_{2, \mu}^{2,1}\left(Q_{T}\right)$ such that $\Phi(0, t)=\varphi(t), \Phi(x, 0)=0$ and

$$
\|\Phi\|_{W_{2, \mu}^{2,1}\left(Q_{T}\right)} \leqslant c\|\varphi\|_{W_{2}^{(1-\mu) / 2}(0, T)} .
$$

Proposition 4.2. (i) If $u \in W_{2, \mu}^{2,1}\left(Q_{T}\right)$, then $u(\cdot, 0) \in W_{2, \mu}^{1}(\Omega)$ and the following estimate holds

$$
\|u(\cdot, 0)\|_{W_{2, \mu}^{1}(\Omega)} \leqslant c\|u\|_{W_{2, \mu}^{2,1}\left(Q_{T}\right)} \cdot
$$

(ii) For any function $u_{0} \in W_{2, \mu}^{1}(\Omega)$ there exists a function $u \in W_{2, \mu}^{2,1}\left(Q_{T}\right)$ such that $u(x, 0)=u_{0}(x)$ and

$$
\|u\|_{W_{2, \mu}^{2,1}\left(Q_{T}\right)} \leqslant c\left\|u_{0}\right\|_{W_{2, \mu}^{1}(\Omega)} \cdot
$$

The following proposition concerns the trace on the surface $\Gamma_{T}=\partial \Omega \times(0, T)$ of $\nabla u$ for a function $u \in W_{2, \mu}^{2,1}\left(Q_{T}\right)$.

Proposition 4.3. (i) If $u \in W_{2, \mu}^{2,1}\left(Q_{T}\right)$, then $\left.\nabla u\right|_{\Gamma_{T}} \in W_{2, \mu}^{1 / 2,1 / 4}\left(\Gamma_{T}\right)$ and the following estimate holds

$$
\left\|\left.\nabla u\right|_{\Gamma_{T}}\right\|_{W_{2, \mu}^{1 / 2,1 / 4}\left(\Gamma_{T}\right)} \leqslant c\|u\|_{W_{2, \mu}^{2,1}\left(Q_{T}\right)} .
$$

(ii) For any function $\phi \in W_{2, \mu}^{1 / 2,1 / 4}\left(\Gamma_{T}\right)$ there exists a function $u \in W_{2, \mu}^{2,1}\left(Q_{T}\right)$ such that $\frac{\partial u}{\partial n}=\phi$ and

$$
\|u\|_{W_{2, \mu}^{2,1}\left(Q_{T}\right)} \leqslant c\|\phi\|_{W_{2, \mu}^{1 / 2,1 / 4}\left(\Gamma_{T}\right)} \cdot
$$

By $\|\phi\|_{W_{2, \mu}^{1 / 2,1 / 4}\left(\Gamma_{T}\right)}$ we mean the norm

$$
\|\phi\|_{W_{2, \mu}^{1 / 2,1 / 4}\left(\Gamma_{T}\right)}=\left\{\int_{0}^{T}\|\phi(\cdot, t)\|_{W_{2, \mu}^{1 / 2}(\partial \Omega)}^{2} d t+\int_{\partial \Omega}\|\phi(x, \cdot)\|_{W_{2}^{1 / 4}(0, T)}^{2}|x|^{2 \mu} d S(x)\right\}^{1 / 2}
$$

where $\|\phi(\cdot, t)\|_{W_{2, \mu}^{1 / 2}(\partial \Omega)}$ is defined as

$$
\|\phi(\cdot, t)\|_{W_{2, \mu}^{1 / 2}(\partial \Omega)}=\left\|\phi|x|^{\mu} \mid\right\|_{W_{2}^{1 / 2}(\partial \Omega)} .
$$

Another equivalent definition is

$$
\|\phi\|_{W_{2, \mu}^{1 / 2}(\partial \Omega)}=\left\{\sum_{i=1}^{2}\|\phi \psi\|_{W_{2, \mu}^{1 / 2}\left(R_{i}\right)}^{2}+\|\phi(1-\psi)\|_{W_{2}^{1 / 2}(\partial \Omega)}\right\}^{1 / 2}
$$


where $\psi=\psi\left(\frac{x}{d_{1}}\right)$ is the same cut-off function that is introduced in Proposition $3.2, R_{i}$ are the two straight sides that constitute the boundary of the infinite angle $\mathscr{D}$ with the vertex $x=0$ and

$$
\|\varphi\|_{W_{2, \mu}^{1 / 2}\left(\mathbb{R}_{+}\right)}^{2}=\|\varphi\|_{L_{2, \mu}}^{2}\left(\mathbb{R}_{+}\right)+\int_{0}^{+\infty} r^{2 \mu} \int_{0}^{r}|\varphi(r+\rho)-\varphi(\rho)|^{2} \rho^{-2} d \rho
$$

$\mathbb{R}_{+}=[0,+\infty)$ (see for instance formula (2.5) in [14]).

Before giving the proof of Propositions 4.1, 4.2 and 4.3 we introduce the spaces $H_{\mu}^{k}(\Omega), H_{\mu}^{2,1}\left(Q_{T}\right)$ and $H_{\mu}^{1,1 / 2}\left(\Gamma_{T}\right)$ of the Kondrat'iev type, (see [21] or [22] where these spaces are denoted by $V_{2, \mu}^{k}(\Omega), V_{2, \mu}^{2,1}\left(Q_{T}\right)$ and $\left.V_{2, \mu}^{1,1 / 2}\left(\Gamma_{T}\right)\right)$ the norms in these spaces coincide with the norms in the spaces $W_{2}^{k}(\Omega), W_{2}^{2,1}\left(Q_{T}\right)$ and $W_{2}^{1,1 / 2}\left(\Gamma_{T}\right)$ of the function multiplied by $|x|^{\mu}$ (as in (4.7)).

We apply to the product $u(x)|x|^{\mu}$ the standard Sobolev-Slobodevskiî trace theorems and obtain (see for instance [35] and [39]):

Proposition 4.4. (i) If $u \in H_{\mu}^{2,1}\left(Q_{T}\right)$, then $u(\cdot, 0) \in H_{\mu}^{1}(\Omega)$ and the following estimate holds

$$
\|u(\cdot, 0)\|_{H_{\mu}^{1}(\Omega)} \leq c\|u\|_{H_{\mu}^{2,1}\left(Q_{T}\right)} .
$$

(ii) If $u \in H_{\mu}^{2,1}\left(Q_{T}\right)$, then $\left.\nabla u\right|_{\Gamma_{T}} \in H_{\mu}^{1 / 2,1 / 4}\left(\Gamma_{T}\right)$ and the following estimate holds

$$
\left\|\left.\nabla u\right|_{\Gamma_{T}}\right\|_{H_{\mu}^{1 / 2,1 / 4}\left(\Gamma_{T}\right)} \leqslant c\|u\|_{H_{\mu}^{2,1}\left(Q_{T}\right)}
$$

Moreover (iii) for any function $u_{0} \in H_{\mu}^{1}(\Omega)$ there exists a function $u \in H_{\mu}^{2,1}\left(Q_{T}\right)$ such that $u(x, 0)=u_{0}(x)$ and

$$
\|u\|_{H_{\mu}^{2,1}\left(Q_{T}\right)} \leqslant c\left\|u_{0}\right\|_{H_{\mu}^{1}(\Omega)}
$$

(iv) For any function $\phi \in H_{\mu}^{1 / 2,1 / 4}\left(\Gamma_{T}\right)$ there exists a function $u \in H_{\mu}^{2,1}\left(Q_{T}\right)$ such that $\frac{\partial u}{\partial n}=\phi, u(\cdot, 0)=0$ and

$$
\|u\|_{H_{\mu}^{2,1}\left(Q_{T}\right)} \leqslant c\|\phi\|_{H_{\mu}^{1 / 2,1 / 4}\left(\Gamma_{T}\right)} .
$$

It is clear that the $H$-spaces are continuously imbedded in the corresponding $W$-spaces. From the Hardy inequalities (that we recall below) it follows that the norms $\|u\|_{W_{2, \mu}^{1}(\Omega)}$ and $\|u\|_{H_{\mu}^{1}(\Omega)}$ are equivalent as well as the norms $\|u\|_{W_{2, \mu}^{2}(\Omega)}$ and $\|u\|_{H_{\mu}^{2}(\Omega)}$ for functions vanishing for $x=0$ and these spaces coincide. In fact for any function $u \in W_{2, \mu}^{1}(\Omega)$ and $\mu \in(0,1)$ we have (see for instance [39])

$$
\|u\|_{L_{2, \mu-1}(\Omega)} \leqslant c\|u\|_{W_{2, \mu}^{1}(\Omega)}
$$


and for $u \in W_{2, \mu}^{2}(\Omega)$ vanishing for $x=0$

$$
\|u\|_{L_{2, \mu-2}(\Omega)} \leqslant c\|u\|_{W_{2, \mu-1}^{1}(\Omega)} \leqslant c\|u\|_{W_{2, \mu}^{2}(\Omega)} .
$$

Now we prove Proposition 4.1.

Proof. To prove inequality (4.1) we extend the function $u \in W_{2, \mu}^{2,1}\left(Q_{T}\right)$ to the infinite time interval $t \in(-\infty,+\infty)$ in such a way that the extended function $u^{*}$ vanishes for $|t| \geqslant T_{0}>T$ and

$$
\left\|u^{*}\right\|_{W_{2, \mu}^{2,1}\left(Q_{\infty}\right)} \leqslant c\|u\|_{W_{2, \mu}^{2,1}\left(Q_{T}\right)}
$$

where $Q_{\infty}=\Omega \times(-\infty,+\infty)$. The extension can be made by reflection with respect to the planes $t=T$ and $t=0$ and subsequent multiplication by an appropriate cut-off function $\zeta(t)$. We set $u_{1}^{*}(x, \cdot)=u^{*}(x, \cdot) \psi\left(\frac{x}{d_{1}}\right)$ where the function $\psi$ is the function used in the proof of Proposition 3.2. We may assume that function $u_{1}^{*}$ is given in the infinite angle $\mathscr{D}$ with the vertex $x=0$ and $u_{1}^{*}(x, \cdot)=0$ for $|x|>d_{1}$; moreover

$$
\left\|u_{1}^{*}\right\|_{W_{2, \mu}^{2,1}\left(\mathscr{D}_{\infty}\right)} \leqslant c\|u\|_{W_{2, \mu}^{2,1}\left(Q_{T}\right)}
$$

where $\mathscr{D}_{\infty}=\mathscr{D} \times(-\infty,+\infty)$. Since $u^{*}(0, \cdot)=u_{1}^{*}(0, \cdot)$ it is enough to prove $(4.1)$ for $u_{1}^{*}$. Let $\hat{u}_{1}^{*}\left(x, \xi_{0}\right)$ denote the Fourier transform of $u_{1}^{*}$ with respect to $t$. Using estimate (A9) in [15] we obtain for arbitrary positive $\rho$

$$
\begin{aligned}
& \left|\hat{u}_{1}^{*}\left(0, \xi_{0}\right)\right|^{2} \\
& \quad \leqslant c\left\{\rho^{2(1-\mu)} \sum_{|\alpha|=2}\left\|D^{\alpha} \hat{u}_{1}^{*}\left(\cdot, \xi_{0}\right)\right\|_{L_{2, \mu}(\mathscr{D})}^{2}+\rho^{2(-1-\mu)}\left\|\hat{u}_{1}^{*}\left(\cdot, \xi_{0}\right)\right\|_{L_{2, \mu}(\mathscr{D})}^{2}\right\} .
\end{aligned}
$$

If we take, in estimate (4.16), $\rho=\left|\xi_{0}\right|^{-1 / 2}$ we obtain

$$
\left|\xi_{0}\right|^{1-\mu}\left|\hat{u}_{1}^{*}\left(0, \xi_{0}\right)\right|^{2} \leqslant c\left\{\sum_{|\alpha|=2}\left\|D^{\alpha} \hat{u}_{1}^{*}\left(\cdot, \xi_{0}\right)\right\|_{L_{2, \mu}(\mathscr{D})}^{2}+\left|\xi_{0}\right|^{2}\left\|\hat{u}_{1}^{*}\left(\cdot, \xi_{0}\right)\right\|_{L_{2, \mu}(\mathscr{D})}^{2}\right\}
$$

if we take, in estimate (4.16), $\rho=1$ we obtain

$$
\left|\hat{u}_{1}^{*}\left(0, \xi_{0}\right)\right|^{2} \leqslant c\left\{\sum_{|\alpha|=2}\left\|D^{\alpha} \hat{u}_{1}^{*}\left(\cdot, \xi_{0}\right)\right\|_{L_{2, \mu}(\mathscr{D})}^{2}+\left\|\hat{u}_{1}^{*}\left(\cdot, \xi_{0}\right)\right\|_{L_{2, \mu}(\mathscr{D})}^{2}\right\} .
$$

Integrating these inequalities with respect to $\xi_{0} \in(-\infty,+\infty)$ and making use of the Parceval identity we obtain

$$
\left\|u^{*}(0, \cdot)\right\|_{W_{2}^{(1-\mu) / 2}(\mathbb{R})}^{2} \leqslant c\left\{\sum_{|\alpha| \leqslant 2}\left\|D^{\alpha} u_{1}^{*}\right\|_{L_{2, \mu}\left(\mathscr{D}_{\infty}\right)}^{2}+\left\|\frac{\partial u_{1}^{*}}{\partial t}\right\|_{L_{2, \mu}\left(\mathscr{D}_{\infty}\right)}^{2}\right\} .
$$


Estimates (4.19) and (4.15) imply

$$
\|u(0, \cdot)\|_{W_{2}^{(1-\mu) / 2}(0, T)} \leqslant c\|u\|_{W_{2, \mu}^{2,1}\left(Q_{T}\right)}
$$

so inequality (4.1) is proved. Now we prove the second statement of Proposition 4.1. We extend the function $\varphi \in W_{2}^{(1-\mu) / 2}(0, T)$ in the time interval $t>T$ (still denoting by $\varphi$ the extended function) so that

$$
\|\varphi\|_{W_{2}^{(1-\mu) / 2}\left(\mathbb{R}_{+}\right)} \leqslant c\|\varphi\|_{W_{2}^{(1-\mu) / 2}(0, T)},
$$

then we extend it by zero in the interval $(-\infty, 0)$. By Lemma 7.1 in [2]

$$
\|\varphi\|_{W_{2}^{(1-\mu) / 2}(\mathbb{R})} \leqslant c\|\varphi\|_{W_{2}^{(1-\mu) / 2}\left(\mathbb{R}_{+}\right)} \leqslant c\|\varphi\|_{W_{2}^{(1-\mu) / 2}(0, T)} .
$$

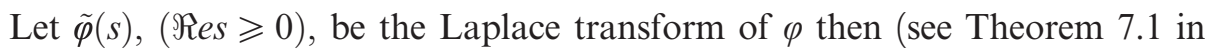
[2]) denoting $\Re$ es by $\sigma$ and $\Im m s$ by $\tau$

$$
\sup _{\sigma>0} \int_{-\infty}^{+\infty}|\tilde{\varphi}(\sigma+i \tau)|^{2}|\sigma+i \tau|^{1-\mu} d \tau \leqslant c\|\varphi\|_{W_{2}^{(1-\mu) / 2}(\mathbb{R})}^{2} \leqslant c\|\varphi\|_{W_{2}^{(1-\mu) / 2}(0, T)}^{2} .
$$

We define $\tilde{\Phi}(x, s)$ in the sector $\mathbb{R}_{+}^{2}:=\left\{x=\left(x_{1}, x_{2}\right): x_{1} \geq 0, x_{2} \geq 0\right\}$ by

$$
\tilde{\Phi}(x, s)=\tilde{\varphi}(s) \exp \left((-\sqrt{s}-1)\left(x_{1}+x_{2}\right)\right),
$$

we note that $\tilde{\Phi}(0, s)=\tilde{\varphi}(s)$ and in the sector $\mathbb{R}_{+}^{2}$ the sum $x_{1}+x_{2}$ is equivalent to $|x|$. The function $\sqrt{s}=|s|^{1 / 2} e^{\text {iargs } / 2}$ (defined for $-\pi<\operatorname{args}<\pi$ ) is holomorphic for $\Re$ es $>0$ and as (for $\Re e s>0$ )

$$
\left|e^{(-\sqrt{s}-1)\left(x_{1}+x_{2}\right)}\right|=e^{(-\Re e \sqrt{s}-1)\left(x_{1}+x_{2}\right)} \leq e^{\left(-c|s|^{1 / 2}-1\right)\left(x_{1}+x_{2}\right)},
$$

we have

$$
\left\|e^{(-\sqrt{s}-1)\left(x_{1}+x_{2}\right)}\right\|_{L_{2, \mu}\left(\mathbb{R}_{+}^{2}\right)} \leq \frac{c}{\left(|s|^{1 / 2}+1\right)^{1+\mu}} .
$$

We then extend the function $\tilde{\Phi}(x, s)$ by using the Hestenes-Whitney formula (see [3], [37] and [20]) in the half plane $x_{1}>0$ and then in the whole domain $\mathscr{D}$.

The extended function $\tilde{\Phi}$ is analytic with respect to $s$ (when $\Re e s>0$ ) so the pre-image (inverse Laplace Transform) of $\tilde{\Phi}$ vanishes for $t \leqslant 0$ (see Theorem 7.1 in [2] or Theorem $\mathrm{V}$ in [33]).

Moreover we deduce from estimate (4.22):

$$
\int_{-\infty}^{+\infty}\left\{|s|^{2}\|\tilde{\Phi}\|_{L_{2, \mu}(\mathscr{D})}^{2}+\|\tilde{\Phi}\|_{W_{2, \mu}^{2}(\mathscr{D})}^{2}\right\} d \tau \leqslant c \int_{-\infty}^{+\infty}|\tilde{\varphi}(s)|^{2}|s|^{1-\mu} d \tau
$$

and for $\Re e s=0$ (see Theorem 8.1 in [2])

$$
\|\Phi\|_{W_{2, \mu}^{2,1}\left(Q_{T}\right)}^{2} \leqslant c \int_{-\infty}^{+\infty}\left\{|\tau|^{2}\|\tilde{\Phi}\|_{L_{2, \mu}(\mathscr{D})}^{2}+\|\tilde{\Phi}\|_{W_{2, \mu}^{2}(\mathscr{D})}^{2}\right\} d \tau
$$


and by formulas (4.21) and (4.23)

$$
\|\Phi\|_{W_{2, \mu}^{2,1}\left(Q_{T}\right)} \leqslant c\|\varphi\|_{W_{2}^{(1-\mu) / 2}(0, T)} .
$$

The proof of Proposition 4.1 is then completed.

We prove now Propositions 4.2 and 4.3.

REMARK 4.1. We have to prove only estimates (4.3) and (4.5) because the second statement of Proposition 4.2 and the second statement of Proposition 4.3 (the inverse trace theorems) follow from the similar statements for $H$-spaces (we mean Proposition 4.4 statements (iii) and (iv) and inequalities (4.10) and (4.11)) (see also Lemma 2.3 in [14]).

Proof of Propositions 4.2. Let $u \in W_{2, \mu}^{2,1}\left(Q_{T}\right)$. Using Proposition 4.1 we construct a function $v \in W_{2, \mu}^{2,1}\left(Q_{T}\right)$ such that $v(0, \cdot)=u(0, \cdot), v(x, 0)=0$ and

$$
\|v\|_{W_{2, \mu}^{2,1}\left(Q_{T}\right)} \leqslant c\|u(0, \cdot)\|_{W_{2}^{(1-\mu) / 2}(0, T)} \leqslant c\|u\|_{W_{2, \mu}^{2,1}\left(Q_{T}\right)} .
$$

Let $w=u-v$, since $w(0, \cdot)=0$ we deduce from (4.24) (see inequalities (4.12), $(4.13))$

$$
\|w\|_{H_{\mu}^{2,1}\left(Q_{T}\right)} \leqslant c\|w\|_{W_{2, \mu}^{2,1}\left(Q_{T}\right)} \leqslant c\|u\|_{W_{2, \mu}^{2,1}\left(Q_{T}\right)}
$$

and by $(4.8)$

$$
\|u(\cdot, 0)\|_{W_{2, \mu}^{1}(\Omega)} \leqslant c\|w(\cdot, 0)\|_{H_{\mu}^{1}(\Omega)} \leqslant c\|w\|_{W_{2, \mu}^{2,1}\left(Q_{T}\right)} \leqslant c\|u\|_{W_{2, \mu}^{2,1}\left(Q_{T}\right)} .
$$

Taking into account Remark 4.1 and inequality (4.26) the proof of Proposition 4.2 is achieved.

Proof of Propositions 4.3. As in Proposition 3.2 we use the splitting

$$
u(x, t)=u(x, t) \psi\left(\frac{x}{d_{1}}\right)+u(x, t)\left(1-\psi\left(\frac{x}{d_{1}}\right)\right):=u_{1}+u_{2} .
$$

The function $u_{2}$ vanishes in a neighborhood of the angular point $x=0$ and for this function estimate (4.5) is a consequence of the similar inequality for the ordinary Sobolev space $W_{2}^{2,1}$ (without weight):

$$
\left\|u_{2}\right\|_{W_{2, \mu}^{1,2,1 / 4}\left(\Gamma_{T}\right)} \leqslant c\left\|u_{2}\right\|_{W_{2}^{1 / 2,1 / 4}\left(\Gamma_{T}\right)} \leqslant c\left\|u_{2}\right\|_{W_{2}^{2,1}\left(Q_{T}\right)} \leqslant c\|u\|_{W_{2, \mu}^{2,1}\left(Q_{T}\right)} .
$$

The function $u_{1}$ vanishes for $|x|>d_{1}$ and it can be extended into the whole $\mathscr{D}$ putting $u_{1}=0$ outside $\Omega$; then we also extend $u_{1}=0$ as previously (see Proposition 4.1) with respect to $t \in(-\infty,+\infty)$. We denote by $u_{1}^{*}$ the extended function. By Theorem 2.2 in [39]

$$
\left\|\nabla u_{1}^{*}(\cdot, t)\right\|_{W_{2, \mu}^{1 / 2}(\partial \mathscr{D})} \leqslant c\left\|\nabla u_{1}^{*}(\cdot, t)\right\|_{W_{2, \mu}^{1}(\mathscr{D})}
$$


and the same inequality holds for the Fourier transform $\hat{u}_{1}^{*}\left(\cdot, \xi_{0}\right)$; in addition we have

$$
\begin{aligned}
& \left\|\nabla \hat{u}_{1}^{*}\left(\cdot, \xi_{0}\right)\right\|_{L_{2, \mu}(\partial \mathscr{D})} \\
& \quad \leqslant c\left\{\left|\xi_{0}\right|^{-1 / 2} \sum_{|\alpha|=2}\left\|D^{\alpha} \hat{u}_{1}^{*}\left(\cdot, \xi_{0}\right)\right\|_{L_{2, \mu}(\mathscr{D})}^{2}+\left|\xi_{0}\right|^{1 / 2}\left\|\nabla \hat{u}_{1}^{*}\left(\cdot, \xi_{0}\right)\right\|_{L_{2, \mu}(\mathscr{D})}^{2}\right\}^{1 / 2} .
\end{aligned}
$$

In fact estimate (4.29) can be obtained by a scaling argument from estimate (7.2) in [39] (that we rewrite for completeness of presentation)

$$
\|\nabla U\|_{L_{2, \mu}(\partial \mathscr{D})} \leqslant c\left\{\sum_{|\alpha|=2}\left\|D^{\alpha} U\right\|_{L_{2, \mu}(\mathscr{D})}^{2}+\|\nabla U\|_{L_{2, \mu}(\mathscr{D})}^{2}\right\}^{1 / 2} .
$$

Hence

$$
\begin{aligned}
& \left\|\nabla \hat{u}_{1}^{*}\left(\cdot, \xi_{0}\right)\right\|_{W_{2, \mu}^{1 / 2}(\partial \mathscr{D})}^{2}+\left|\xi_{0}\right|^{1 / 2}\left\|\nabla \hat{u}_{1}^{*}\left(\cdot, \xi_{0}\right)\right\|_{L_{2, \mu}(\partial \mathscr{D})}^{2} \\
& \quad \leqslant c\left\{\sum_{|\alpha| \leqslant 2}\left\|D^{\alpha} \hat{u}_{1}^{*}\left(\cdot, \xi_{0}\right)\right\|_{L_{2, \mu}(\mathscr{D})}^{2}+\left|\xi_{0}\right|\left\|\nabla \hat{u}_{1}^{*}\left(\cdot, \xi_{0}\right)\right\|_{L_{2, \mu}(\mathscr{D})}^{2}\right\} .
\end{aligned}
$$

By Remark 3.1 the right hand side in (4.31) does not exceed

$$
c\left\{\sum_{|\alpha| \leqslant 2}\left\|D^{\alpha} \hat{u}_{1}^{*}\left(\cdot, \xi_{0}\right)\right\|_{L_{2, \mu}(\mathscr{D})}^{2}+\left|\xi_{0}\right|^{2}\left\|\hat{u}_{1}^{*}\left(\cdot, \xi_{0}\right)\right\|_{L_{2, \mu}(\mathscr{D})}^{2}\right\} .
$$

After integration with respect to $\xi_{0} \in \mathbb{R}$ we obtain

$$
\left\|u_{1}\right\|_{W_{2, \mu}^{1 / 2,1 / 4}\left(\Gamma_{T}\right)} \leqslant c\left\|u_{1}^{*}\right\|_{W_{2, \mu}^{1 / 2,1 / 4}\left(\mathscr{D}_{\infty}\right)} \leqslant c\left\|u_{1}\right\|_{W_{2, \mu}^{2,1}\left(Q_{T}\right)} \leqslant c\|u\|_{W_{2, \mu}^{2,1}\left(Q_{T}\right)} .
$$

Together with (4.27) inequality (4.32) yields (4.5).

\section{The parabolic Neumann problem}

In this section we establish existence, uniqueness results and coercive estimates for the solution of the parabolic Neumann problem

$$
\begin{gathered}
\frac{\partial v}{\partial t}+L v=d(x, t) \quad x \in \Omega \subset \mathbb{R}^{2}, t \in(0, T) \\
\left.\frac{\partial v}{\partial n}\right|_{x \in \partial \Omega}=0, \quad v(x, 0)=v_{0}(x)
\end{gathered}
$$


and the corresponding parameter-dependent problem

$$
\begin{gathered}
\lambda u+L u=f(x) \quad x \in \Omega \subset \mathbb{R}^{2}, \\
\left.\frac{\partial u}{\partial n}\right|_{x \in \partial \Omega}=0,
\end{gathered}
$$

with a complex-value parameter $\lambda$; the functions $f, u$ are also complex-valued. Here $L=-\triangle+1, \triangle$ is the Laplace operator and we assume that $\Omega$ is a bounded domain whose boundary $\partial \Omega$ is smooth everywhere except for a singular angular point $x=0$. The opening of the angle denoted by $\theta$ is supposed to be greater than $\pi$.

The main results of this section are the following

THEOREM 5.1. If $d \in L_{2, \mu}\left(Q_{T}\right), v_{0} \in W_{2, \mu}^{1}(\Omega), 1-\pi / \theta<\mu<1$ then problem (5.1) has a unique solution $v \in W_{2, \mu}^{2,1}\left(Q_{T}\right)$, and $v(x, t)$ satisfies the inequality

$$
\|v\|_{W_{2, \mu}^{2,1}\left(Q_{T}\right)} \leq c\left\{\|d\|_{L_{2, \mu}\left(Q_{T}\right)}+\left\|v_{0}\right\|_{W_{2, \mu}^{1}(\Omega)}\right\}
$$

where the constant $c$ is independent of $T$.

THEOREM 5.2. Let $f \in L_{2, \mu}(\Omega), 1-\pi / \theta<\mu<1$ and $\lambda$ satisfy the condition

$$
\Re e \lambda>\min \{-\gamma,-x|\Im m \lambda|\}
$$

with $\gamma, x \in(0,1)$. Then problem (5.2) has a unique solution $u \in W_{2, \mu}^{2}(\Omega)$, and $u(x)$ satisfies the inequality

$$
\|u\|_{W_{2, \mu}^{2}(\Omega)}+(1+|\lambda|)\|u\|_{L_{2, \mu}(\Omega)} \leq c\|f\|_{L_{2, \mu}(\Omega)}
$$

where the constant $c$ is independent of $\lambda$, but it may depend on $\gamma$ and $\chi$.

We start by proving Theorem 5.2.

ProOF. We say that a function $u \in W_{2}^{1}(\Omega)$ is a weak solution of problem (5.2) if $u$ satisfies the integral identity

$$
Q_{\lambda}(u, \varphi):=(1+\lambda) \int_{\Omega} u \bar{\varphi} d x+\int_{\Omega} \nabla u \nabla \bar{\varphi} d x=\int_{\Omega} f \bar{\varphi} d x
$$

for any $\varphi \in W_{2}^{1}(\Omega)$ (all the functions are complex-valued and $\nabla u \nabla \bar{\varphi}=$ $\left.\sum_{i=1}^{2} u_{x_{i}} \bar{\varphi}_{x_{i}}\right)$.

We show that for arbitrary $f \in L_{2, \mu}(\Omega)$ there exists a unique weak solution via the Lax-Milgram theorem because the form $Q_{\lambda}$ possesses the property:

$$
\left|Q_{\lambda}(u, u)\right| \geq c(1+|\lambda|)\|u\|_{L_{2}(\Omega)}^{2}+1 / 2\|\nabla u\|_{L_{2}(\Omega)}^{2}
$$


if $\lambda$ satisfies condition (5.4). In fact

$$
\Re e Q_{\lambda}(u, u)+\left|\Im m Q_{\lambda}(u, u)\right|=(\Re e \lambda+|\Im m \lambda|+1)\|u\|_{L_{2}(\Omega)}^{2}+\|\nabla u\|_{L_{2}(\Omega)}^{2}
$$

and if condition (5.4) holds then

$$
(\Re e \lambda+|\Im m \lambda|+1) \geq c(|\lambda|+1) .
$$

Hence

$$
\Re e Q_{\lambda}(u, u)+\left|\Im m Q_{\lambda}(u, u)\right| \geq c(|\lambda|+1)\|u\|_{L_{2}(\Omega)}^{2}+\|\nabla u\|_{L_{2}(\Omega)}^{2}
$$

and

$$
\begin{aligned}
\left|Q_{\lambda}(u, u)\right| & \geq 1 / 2\left(\Re e Q_{\lambda}(u, u)+\left|\Im m Q_{\lambda}(u, u)\right|\right) \\
& \geq 1 / 2\left\{c(|\lambda|+1)\|u\|_{L_{2}(\Omega)}^{2}+\|\nabla u\|_{L_{2}(\Omega)}^{2}\right\} .
\end{aligned}
$$

Setting $\varphi=u$ in (5.6) we obtain

$$
\left|Q_{\lambda}(u, u)\right| \leq\|f\|_{L_{2, \mu}(\Omega)}\left(\int_{\Omega} u^{2}|x|^{-2 \mu} d x\right)^{1 / 2} .
$$

Now to evaluate the last term in inequality (5.9) we use estimate (4.11) in [39] where we put $\xi=(1+|\lambda|)^{1 / 2}$. This gives

$$
(|\lambda|+1)^{1-\mu} \int_{\Omega} u^{2}|x|^{-2 \mu} d x \leq c\left\{(|\lambda|+1)\|u\|_{L_{2}(\Omega)}^{2}+\|\nabla u\|_{L_{2}(\Omega)}^{2}\right\} .
$$

Finally inequalities (5.8), (5.9) and (5.10) yield

$$
(|\lambda|+1)^{1-\mu}\left\{(|\lambda|+1)\|u\|_{L_{2}(\Omega)}^{2}+\|\nabla u\|_{L_{2}(\Omega)}^{2}\right\} \leq c\|f\|_{L_{2, \mu}(\Omega)}^{2} .
$$

Next step is to prove the inequality

$$
(|\lambda|+1)\left\{(|\lambda|+1)\|u\|_{L_{2, \mu}(\Omega)}^{2}+\|\nabla u\|_{L_{2, \mu}(\Omega)}^{2}\right\} \leq c\|f\|_{L_{2, \mu}(\Omega)}^{2} .
$$

We set $\varphi=u|x|^{2 \mu}$ in (5.6) and we repeat the previous arguments to obtain

$$
\begin{aligned}
(|\lambda|+1)\|u\|_{L_{2, \mu}(\Omega)}^{2}+\|\nabla u\|_{L_{2, \mu}(\Omega)}^{2} & \\
& \leq c\left\{\int_{\Omega}|f||u||x|^{2 \mu} d x+\int_{\Omega}|\nabla u||u||x|^{2 \mu-1} d x\right\} .
\end{aligned}
$$

By Cauchy inequality we have

$$
\int_{\Omega}|\nabla u||u||x|^{2 \mu-1} d x \leq \epsilon \int_{\Omega}|\nabla u|^{2}|x|^{2 \mu} d x+c / \epsilon \int_{\Omega}|u|^{2}|x|^{2 \mu-2} d x
$$


where we choose $\epsilon \ll 1$. To evaluate the last integral in the previous inequality we again use estimate (4.11) in [39] where now we replace $\mu$ by $1-\mu$ and put $\xi=(1+|\lambda|)^{1 / 2}$. This gives, using also estimate (5.11)

$$
\begin{aligned}
\int_{\Omega}|u|^{2}|x|^{2 \mu-2} d x & \leq c(|\lambda|+1)^{-\mu} \int_{\Omega}\left\{|\nabla u|^{2}+(|\lambda|+1)|u|^{2}\right\} d x \\
& \leq c(|\lambda|+1)^{-1}\|f\|_{L_{2, \mu}(\Omega)}^{2} .
\end{aligned}
$$

Another term in (5.13) is estimated as follows

$$
\begin{aligned}
\int_{\Omega}|u||f||x|^{2 \mu} d x \leq & \left\{(|\lambda|+1) \int_{\Omega} u^{2}|x|^{2 \mu} d x\right\}^{1 / 2} \\
& \cdot\left\{(|\lambda|+1)^{-1} \int_{\Omega} f^{2}|x|^{2 \mu} d x\right\}^{1 / 2} .
\end{aligned}
$$

From (5.13) (5.14) and (5.15) we obtain

$$
(|\lambda|+1) \int_{\Omega} u^{2}|x|^{2 \mu} d x+\int_{\Omega}|\nabla u|^{2}|x|^{2 \mu} d x \leq c(|\lambda|+1)^{-1} \int_{\Omega} f^{2}|x|^{2 \mu} d x
$$

and this estimate (5.16) is equivalent to (5.12).

To evaluate the second derivatives of the function $u$, we consider $u$ as weak solution of the Neumann problem for the equation

$$
\triangle u=-f(x)+(\lambda+1) u
$$

$u$ satisfies the integral identity

$$
\int_{\Omega} \nabla u \nabla \bar{\varphi} d x=\int_{\Omega}\{f-(1+\lambda) u\} \bar{\varphi} d x
$$

for any $\varphi \in W_{2}^{1}(\Omega)$ with $f-(1+\lambda) u \in L_{2, \mu}(\Omega)$. From the results of $\mathrm{V}$. A. Kondrat'iev (see [21]) it follows that $D^{\alpha} u \in L_{2, \mu}(\Omega)$ for $|\alpha|=2$ and

$$
\left\|D^{\alpha} u\right\|_{L_{2, \mu}(\Omega)} \leq c\|f-(1+\lambda) u\|_{L_{2, \mu}(\Omega)} \leqslant c\|f\|_{L_{2, \mu}(\Omega)}
$$

Together with (5.12) this inequality yields (5.5) and the proof of Theorem 5.2 is now complete.

Proof of Theorem 5.1. We reduce Problem (5.1) to a similar problem with zero initial data. Using Proposition 4.2, Proposition 4.1 and Proposition 4.4, we construct the function $w \in W_{2, \mu}^{2,1}\left(Q_{T}\right)$ such that $w(x, 0)=v_{0}(x)$ for $x \in \Omega, \frac{\partial w}{\partial n}=0$ for $x \in \partial \Omega$ and

$$
\|w\|_{W_{2, \mu}^{2,1}\left(Q_{T}\right)} \leq c\left\|v_{0}\right\|_{W_{2, \mu}^{1}(\Omega)} \cdot
$$


The difference $u=v-w$ is solution of the problem

$$
\begin{gathered}
\frac{\partial u}{\partial t}+L u=f(x, t) \quad x \in \Omega \subset \mathbb{R}^{2}, t \in(0, T) \\
\left.\frac{\partial u}{\partial n}\right|_{x \in \partial \Omega}=0, \quad u(x, 0)=0
\end{gathered}
$$

where $f=d-\frac{\partial w}{\partial t}-L w \in L_{2, \mu}\left(Q_{T}\right)$ and

$$
\|f\|_{L_{2, \mu}\left(Q_{T}\right)} \leq\|d\|_{L_{2, \mu}\left(Q_{T}\right)}+\|w\|_{W_{2, \mu}^{2,1}\left(Q_{T}\right)} \leq c\left(\|d\|_{L_{2, \mu}\left(Q_{T}\right)}+\left\|v_{0}\right\|_{W_{2, \mu}^{1}(\Omega)}\right) .
$$

We solve problem (5.18) by the Laplace transform as in [2]. We introduce the extension of $f$ on $\Omega \times \mathbb{R}$ denoted by $f$ and defined as follows: we first extend it into the time interval $t \in(T, 2 T)$ by reflection, then it is multiplied by an appropriate cut-off function of $t$ and finally we set $f=0$ for $t<0$. Clearly the extended function satisfies the inequality

$$
\|f\|_{L_{2, \mu}(\Omega \times \mathbb{R})} \leq c\|f\|_{L_{2, \mu}\left(Q_{T}\right)} .
$$

The Laplace transform converts problem (5.18) in

$$
\begin{gathered}
s \tilde{u}+L \tilde{u}=\tilde{f} \quad x \in \Omega \subset \mathbb{R}^{2}, \\
\left.\frac{\partial \tilde{u}}{\partial n}\right|_{x \in \partial \Omega}=0 .
\end{gathered}
$$

We assume that $\Re e s \geqslant 0$ and from Theorem 5.2 we deduce the existence of the unique solution of problem (5.21) and the estimate

$$
(|s|+1)\|\tilde{u}\|_{L_{2, \mu}(\Omega)}^{2}+\|\tilde{u}\|_{W_{2, \mu}^{2}(\Omega)}^{2} \leq c\|\tilde{f}\|_{L_{2, \mu}(\Omega)} .
$$

The inverse Laplace transform yields the solution of problem (5.18) that is unique. Setting $\Re e s=0$ and integrating with respect to $\Im m s$ in the line $(-\infty,+\infty)$ we obtain

$$
\|u\|_{W_{2, \mu}^{2,1}\left(Q_{T}\right)}^{2} \leq c\|f\|_{L_{2, \mu}\left(Q_{T}\right)}^{2} .
$$

Together with (5.17) and (5.19) this inequality yields (5.3). The proof of Theorem 5.1 is complete.

\section{THE LINEAR PROBLEM}

In this section we consider the linear initial-boundary value problem

$$
\begin{gathered}
\frac{\partial u}{\partial t}+A\left(x, \frac{\partial}{\partial x}\right) u=f(x, t) \quad x \in \Omega \subset \mathbb{R}^{2}, t \in(0, T) \\
\left.\frac{\partial v}{\partial n}\right|_{x \in \partial \Omega}=0, \quad u(x, 0)=u_{0}(x)
\end{gathered}
$$


where $u(x, t)=(v(x, t), w(x, t)), u_{0}=\left(v_{0}, w_{0}\right), f(x, t)=(g(x, t), h(x, t)), A$ is a matrix differential operator of the form (1.2), i.e.

$$
A=\left(\begin{array}{cc}
L & \ell_{1}(x) \\
\ell_{2}(x) & \ell_{3}(x)
\end{array}\right),
$$

$L=-\triangle+1, \triangle$ is the Laplace operator and $\ell_{1}(x), \ell_{2}(x), \ell_{3}(x)$ are real-valued functions given in $\Omega$. We assume that $\Omega$ is a bounded domain $\left(\Omega \subset \mathbb{R}^{2}\right)$ and $\partial \Omega$ is smooth everywhere except for a singular angular point $x=0$. In a neighborhood of this point, the boundary of $\Omega$ consists of two straight lines. The opening of the angle denoted by $\theta$ is supposed to be greater than $\pi$.

Our objective is to establish existence, uniqueness results and coercive estimates for the solution of the problem (6.1). These estimates, which in our opinion are of interest in themselves, are crucial for proving the stability result for nonlinear problem (1.1) (see Theorem 7.1).

The first result of this section is

TheOREM 6.1. If $\ell_{1} \in L_{\infty}(\Omega), \ell_{2}, \ell_{3} \in W_{2}^{s}(\Omega), \quad v_{0} \in W_{2, \mu}^{1}(\Omega), g \in L_{2, \mu}\left(Q_{T}\right)$, $w_{0} \in W_{2}^{s}(\Omega), h \in W_{2}^{s, 0}\left(Q_{T}\right), 1-\pi / \theta<\mu<1,1<s<2-\mu$, then problem $(6.1)$ has a unique solution $u=(v, w)$ with $v \in W_{2, \mu}^{2,1}\left(Q_{T}\right), w, \frac{\partial w}{\partial t} \in W_{2}^{s, 0}\left(Q_{T}\right)$, and the following estimate holds:

$$
\begin{aligned}
& \|v\|_{W_{2, \mu}^{2,1}\left(Q_{T}\right)}+\|w\|_{W_{2}^{s, 0}\left(Q_{T}\right)}+\left\|\frac{\partial w}{\partial t}\right\|_{W_{2}^{s, 0}\left(Q_{T}\right)} \\
& \quad \leq c(T)\left\{\|g\|_{L_{2, \mu}\left(Q_{T}\right)}+\|h\|_{W_{2}^{s, 0}\left(Q_{T}\right)}+\left\|v_{0}\right\|_{W_{2, \mu}^{1}(\Omega)}+\left\|w_{0}\right\|_{W_{2}^{s}(\Omega)}\right\} .
\end{aligned}
$$

The proof of Theorem 6.1 is based on the analysis of the Cauchy problem

$$
\frac{\partial w}{\partial t}(x, t)+\ell_{3}(x) w(x, t)=\varphi(x, t),\left.\quad w\right|_{t=0}=w_{0}(x), \quad x \in \Omega, t \in(0, T),
$$

and of the parabolic initial-boundary value problem (5.1).

We start by proving the following proposition

Proposition 6.1. If $w_{0} \in W_{2}^{s}(\Omega), \ell_{3} \in W_{2}^{s}(\Omega)$ and $\varphi \in W_{2}^{s, 0}\left(Q_{T}\right), 1<s$, then problem (6.3) has a unique solution $w \in W_{2}^{s, 0}\left(Q_{T}\right)$, moreover the time derivative $\frac{\partial w}{\partial t}$ belongs to the space $W_{2}^{s, 0}\left(Q_{T}\right)$ and

$$
\begin{gathered}
\|w\|_{W_{2}^{s, 0}\left(Q_{T}\right)}+\left\|\frac{\partial w}{\partial t}\right\|_{W_{2}^{s, 0}\left(Q_{T}\right)}+\sup _{t \in(0, T)}\|w(\cdot, t)\|_{W_{2}^{s}(\Omega)} \\
\leq c(T)\left(\left\|w_{0}\right\|_{W_{2}^{s}(\Omega)}+\|\varphi\|_{W_{2}^{s, 0}\left(Q_{T}\right)}\right) .
\end{gathered}
$$


ProOF. It is well known that the solution of the problem (6.3) is given by the formula

$$
w(x, t)=V(t) w_{0}(x)+\int_{0}^{t} V(t-\tau) \varphi(x, \tau) d \tau,
$$

where $V(t)=e^{-t / 3}$ is the resolving operator of problem (6.3).

Estimate (6.4) follows easily from (6.5) by using the Young inequality for convolutions (see e.g. [4]), because

$$
\|V(t) w\|_{W_{2}^{s}(\Omega)} \leq c(T)\|w\|_{W_{2}^{s}(\Omega)} .
$$

Proposition 6.1 is proved; let us note that in general, $c(T)$ grows exponentially, as $T \rightarrow+\infty$.

Proof of TheOrem 6.1. We start by the reduction of the problem (6.1) to the problem (5.1) with an additional non-local lower order term in the equation for $v$. We consider $w$ as a solution of (6.3) with $\varphi=h-\ell_{2}(x) v$, which yields the following expression for $w$ :

$$
w(x, t)=V(t) w_{0}(x)+\int_{0}^{t} V(t-\tau) h(x, \tau) d \tau-\int_{0}^{t} V(t-\tau) \ell_{2}(x) v(x, \tau) d \tau .
$$

When we plug this expression into the first equation of the system (6.1), we obtain the initial-boundary value problem for $v$ :

where

$$
\begin{gathered}
\frac{\partial v}{\partial t}(x, t)+L v(x, t)-\ell_{1}(x) \int_{0}^{t} V(t-\tau) \ell_{2}(x) v(x, \tau) d \tau \\
=d(x, t), \quad(x, t) \in Q_{T}, \\
\left.\frac{\partial v}{\partial n}\right|_{x \in \partial \Omega}=0, \quad v(x, 0)=v_{0}(x),
\end{gathered}
$$

$$
d(x, t) \equiv g(x, t)-\ell_{1}(x)\left(V(t) w_{0}(x)+\int_{0}^{t} V(t-\tau) h(x, \tau) d \tau\right)
$$

It is easily shown that

$$
\|d\|_{L_{2, \mu}\left(Q_{T}\right)} \leq\|g\|_{L_{2, \mu}\left(Q_{T}\right)}+c(T)\left(\left\|w_{0}\right\|_{L_{2, \mu}(\Omega)}+\|h\|_{L_{2, \mu}\left(Q_{T}\right)}\right) .
$$

Problem (6.7) differs from (5.1) by the presence of the integral operator of the Volterra type

$$
\mathscr{I} v=-\ell_{1}(x) \int_{0}^{t} V(t-\tau) \ell_{2}(x) v(x, \tau) d \tau
$$


in the equation. It satisfies the inequality

$$
\|\mathscr{I} v\|_{L_{2, \mu}\left(Q_{t}\right)} \leq c(T)\|v\|_{L_{2, \mu}\left(Q_{t}\right)}
$$

that allows us to solve problem (6.7) by successive approximations, according to the following scheme.

We define $v_{1}$ as the solution of the problem

$$
\begin{gathered}
\frac{\partial v_{1}}{\partial t}+L v_{1}=d(x, t), \quad x \in \Omega, t \in(0, T), \\
\left.\frac{\partial v_{1}}{\partial n}\right|_{x \in \partial \Omega}=0, \quad v_{1}(x, 0)=v_{0}(x)
\end{gathered}
$$

and we find $v_{m+1}$ from

$$
\begin{gathered}
\frac{\partial v_{m+1}}{\partial t}+L v_{m+1}=\ell_{1}(x) \int_{0}^{t} V(t-\tau) \ell_{2}(x) v_{m}(x, \tau) d \tau+d(x, t), \quad x \in \Omega, t \in(0, T), \\
\left.\frac{\partial v_{m+1}}{\partial n}\right|_{x \in \partial \Omega}=0, \quad v_{m+1}(x, 0)=v_{0}(x) .
\end{gathered}
$$

Set

$$
\xi_{m+1}=v_{m+1}-v_{m}, \quad m \geq 1
$$

and

$$
\xi_{1}=v_{1}
$$

The function $\xi_{m+1}, m \geq 1$, is a solution of the problem

$$
\begin{gathered}
\frac{\partial \xi_{m+1}}{\partial t}+L \xi_{m+1}=-\mathscr{I} \xi_{m}, \quad x \in \Omega, t \in(0, T), \\
\left.\frac{\partial \xi_{m+1}}{\partial n}\right|_{x \in \partial \Omega}=0, \quad \xi_{m+1}(x, 0)=0 .
\end{gathered}
$$

From Theorem 5.1 (see (5.3)) and (6.11) we obtain

$$
\left\|\xi_{m+1}\right\|_{W_{2, \mu}^{2,1}\left(Q_{t}\right)} \leq c(T)\left\|\xi_{m}\right\|_{L_{2, \mu}\left(Q_{t}\right)}, \quad m \geq 1
$$

which implies

$$
\sum_{m=0}^{M}\left\|\xi_{m+1}\right\|_{W_{2, \mu}^{2,1}\left(Q_{t}\right)} \leq c(T)\left(\left\|\xi_{1}\right\|_{W_{2, \mu}^{2,1}\left(Q_{t}\right)}+\sum_{m=1}^{M}\left\|\xi_{m+1}\right\|_{L_{2, \mu}\left(Q_{t}\right)}\right)
$$


The last sum in the right hand side does not exceed

$$
\begin{aligned}
& \sum_{m=1}^{M} \sup _{\tau<t}\left\|\xi_{m+1}(\cdot, \tau)\right\|_{L_{2, \mu}(\Omega)}^{1 / 2}\left(\int_{0}^{t}\left\|\xi_{m+1}\right\|_{L_{2, \mu}(\Omega)} d \tau\right)^{1 / 2} \\
& \quad \leq T^{1 / 4}\left(\sum_{m=1}^{M}\left\|\frac{\partial \xi_{m+1}}{\partial \tau}\right\|_{L_{2, \mu}\left(Q_{t}\right)}\right)^{1 / 2}\left(\sum_{m=1}^{M} \int_{0}^{t}\left\|\xi_{m+1}\right\|_{L_{2, \mu}(\Omega)} d \tau\right)^{1 / 2} .
\end{aligned}
$$

Hence applying the Cauchy inequality we obtain

$$
\begin{aligned}
\sum_{m=0}^{M}\left\|\xi_{m+1}\right\|_{W_{2, \mu}^{2,1}\left(Q_{t}\right)} & \leq c(T)\left(\left\|\xi_{1}\right\|_{W_{2, \mu}^{2,1}\left(Q_{t}\right)}+\sum_{m=1}^{M} \int_{0}^{t}\left\|\xi_{m+1}\right\|_{L_{2, \mu}(\Omega)} d \tau\right) \\
& \leq c(T)\left(\left\|\xi_{1}\right\|_{W_{2, \mu}^{2,1}\left(Q_{t}\right)}+\int_{0}^{t} \sum_{m=0}^{M}\left\|\xi_{m+1}\right\|_{W_{2, \mu}^{2,1}\left(Q_{\tau}\right)} d \tau\right) .
\end{aligned}
$$

Finally, using the Gronwall lemma, we arrive at

$$
\sum_{m=0}^{M}\left\|\xi_{m+1}\right\|_{W_{2, \mu}^{2,1}\left(Q_{T}\right)} \leq c(T)\left\|\xi_{1}\right\|_{W_{2, \mu}^{2,1}\left(Q_{T}\right)} \leq c(T)\left(\|d\|_{L_{2, \mu}\left(Q_{T}\right)}+\left\|v_{0}\right\|_{W_{2, \mu}^{1}(\Omega)}\right)
$$

This shows that the sequence $\left\{v_{m}\right\}$ is convergent in the Sobolev space $W_{2, \mu}^{2,1}\left(Q_{T}\right)$ to a solution of problem (6.7).

The uniqueness of the solution follows from inequalities (5.3) and (6.11) applied to the difference of two solutions $v$ and $v^{\prime}$ of (6.7). Since

$$
\left\|v-v^{\prime}\right\|_{W_{2, \mu}^{2,1}\left(Q_{t}\right)} \leq c(T) t\left\|v-v^{\prime}\right\|_{W_{2, \mu}^{2,1}\left(Q_{t}\right)},
$$

there exits a positive time $T_{0}$ such that $v=v^{\prime}$ for $t<T_{0}$. In a finite number of steps we prove that $v=v^{\prime}$ for $t \in(0, T)$.

Consequently also problem (6.1) admits a unique solution. By (6.12), (6.9)

$$
\|v\|_{W_{2, \mu}^{2,1}\left(Q_{T}\right)} \leq c(T)\left(\|g\|_{L_{2, \mu}\left(Q_{T}\right)}+\left\|v_{0}\right\|_{W_{2, \mu}^{1}(\Omega)}+\left\|w_{0}\right\|_{W_{2}^{s}(\Omega)}+\|h\|_{W_{2}^{s, 0}\left(Q_{T}\right)}\right) .
$$

Inequality (6.2) follows from estimates (6.13) and (6.4). Theorem 6.1 is proved.

Theorem 6.1 allows us to define the operator $e^{-A t}$ and write the solution of (6.1) in the form

$$
u=e^{-A t} u_{0}+\int_{0}^{t} e^{-A(t-\tau)} f(\cdot, \tau) d \tau
$$

where $u_{0}=\left(v_{0}, w_{0}\right) \in W_{2, \mu}^{1}(\Omega) \times W_{2}^{s}(\Omega), f(x, t)=(g(x, t), h(x, t)) \in L_{2, \mu}\left(Q_{T}\right) \times$ $W_{2}^{s, 0}\left(Q_{T}\right)$. 
Assuming suitable conditions on the spectrum of the operator $-A$ and on the coefficient $\ell_{3}$ we establish the exponential decay of the operator $e^{-A t}$. More precisely we denote

$$
X:=L_{2, \mu}(\Omega) \times W_{2}^{s}(\Omega)
$$

then the following result holds.

THEOREM 6.2. We assume that $\ell_{1} \in L_{\infty}(\Omega), \ell_{2}, \ell_{3} \in W_{2}^{s}(\Omega), v_{0} \in W_{2, \mu}^{1}(\Omega), g \in$ $L_{2, \mu}\left(Q_{T}\right), w_{0} \in W_{2}^{s}(\Omega), h \in W_{2}^{s, 0}\left(Q_{T}\right), 1-\pi / \theta<\mu<1,1<s<2-\mu$. Moreover we suppose that the following conditions are satisfied

a) $\ell_{3}(x) \geq b_{1}>0$,

b) the spectrum of the operator $-A$ is located in the half-plane $\Re e \lambda<-b_{1}$.

Then

$$
\left\|e^{-A t}\right\|_{X} \leq c e^{-\beta_{1} t}
$$

where $\beta_{1}<b_{1}$ and $X$ is defined in (6.14). As a consequence

$$
\left\|e^{\beta t} u\right\|_{L_{2}(0, T ; X)} \leq c\left(\left\|u_{0}\right\|_{X}+\left\|e^{\beta t} f\right\|_{L_{2}(0, T ; X)}\right)
$$

where $\beta<\beta_{1}$ and the constant $c$ does not depend on $T$.

PROOF. We prove inequality $(6.15)$ by using the resolvent estimate. We consider the following parameter-dependent problem where $\hat{u}=(\hat{v}, \hat{w}), \hat{f}=(\hat{g}, \hat{h}), \lambda \in \mathbb{C}$ :

$$
\left\{\begin{array}{l}
\lambda \hat{u}+A \hat{u}=\hat{f} \in X \\
\left.\frac{\partial \hat{v}}{\partial n}\right|_{x \in \partial \Omega}=0,
\end{array}\right.
$$

and we prove that there exists such positive $\varkappa$ that this problem is uniquely solvable for arbitrary $\hat{f} \in X$ and for any $\lambda$ in the set

$$
\mathfrak{C}_{\varkappa, \beta_{1}}=\left\{\mathscr{R} e \lambda \geq \min \left\{-\beta_{1},-\chi|\mathscr{I} m \lambda|\right\}\right\} ; \quad \beta_{1}<b_{1} .
$$

Moreover, the solution satisfies the inequality

$$
(|\lambda|+1)\|\hat{u}\|_{X}+\|A \hat{u}\|_{X} \leq C\|\hat{f}\|_{X}
$$

which implies

$$
(|\lambda|+1)\|\hat{u}\|_{X}+\|\hat{v}\|_{W_{2, \mu}^{2}(\Omega)}+\|\hat{w}\|_{W_{2}^{s}(\Omega)} \leq C\|\hat{f}\|_{X}
$$

The constants in these inequalities are independent of $\lambda$, but they may depend on $x$ and $\beta_{1}$. 
Let $\Re \subset \mathbb{R}_{-}$denote the closure (in $\mathbb{R}$ ) of the range of the function $-\ell_{3}(x)$; it is clear that for any $\beta_{1}<b_{1}, \Re \cap \mathfrak{C}_{\varkappa, \beta_{1}}=\emptyset$.

If $\lambda$ is not in $\Re$, then the equation

$$
\lambda \hat{w}+\ell_{2} \hat{v}+\ell_{3} \hat{w}=\hat{h}
$$

has a unique solution

$$
\hat{w}=\frac{\hat{h}-\ell_{2}(x) \hat{v}}{\lambda+\ell_{3}(x)} .
$$

We plug (6.20) in the first equation in (6.17) and we obtain

$$
\left\{\begin{array}{l}
\lambda \hat{v}+L \hat{v}=\psi \\
\left.\frac{\partial \hat{v}}{\partial n}\right|_{x \in \partial \Omega}=0 .
\end{array}\right.
$$

where

$$
\psi=\hat{g}-\ell_{1}(x) \frac{\hat{h}-\ell_{2}(x) \hat{v}}{\lambda+\ell_{3}(x)} .
$$

We consider at first problem (6.21) with a given $\psi \in L_{2, \mu}(\Omega)$. It is uniquely solvable for all $\lambda$ such that $\mathscr{R} e \lambda \geq-\chi|\mathscr{I} m \lambda|,(x \in(0,1))$ (in fact, for all $\lambda$ outside the half-axis $\Re e \lambda \leqslant-1, \Im m \lambda=0)$, and the solution satisfies the inequality

$$
\begin{aligned}
(|\lambda|+1)\|\hat{v}\|_{L_{2, \mu}(\Omega)}+\|\hat{v}\|_{W_{2, \mu}^{2}(\Omega)} & \leq c\left((|\lambda|+1)\|\hat{v}\|_{L_{2, \mu}(\Omega)}+\|L \hat{v}\|_{L_{2, \mu}(\Omega)}\right) \\
& \leq c_{0}\|\psi\|_{L_{2, \mu}(\Omega)} .
\end{aligned}
$$

This statement is proved in Section 5 for $\varkappa \in(0,1)$ (see Theorem 5.2). By using the contraction mapping theorem we can prove that problem $(6.21),(6.22)$ is also uniquely solvable and the inequality (6.18) is satisfied for

$$
\lambda \in \Sigma_{\varkappa, \rho}=\{\operatorname{Re} \lambda \geq-\varkappa|\operatorname{Im} \lambda|,|\lambda| \geq \rho\}
$$

with sufficiently large $\rho$. We stress the fact that the lower bound of $\rho$ depends only on the the constant $c_{0}$ in (6.23) and on the $L_{\infty}$-norms of the data $\ell_{1}, \ell_{2}, \ell_{3}$. It is easily seen that $\hat{f}=0$ implies $\hat{u}=0$.

Now we consider the equations (6.21), (6.22) for arbitrary $\lambda \in \mathbb{C} \backslash \Re$. We choose a (real) number $a>\rho$ such that the operator $L+a I$ has the inverse $(L+a I)^{-1}$ and we write these equations in the form

$$
\hat{v}-\mathscr{L}(v) \hat{v}=(L+a I)^{-1}\left(-\ell_{1} \hat{h}\left(\ell_{3}+(v+a)\right)^{-1}+\hat{g}\right)
$$

where $v=\lambda-a$,

$$
\mathscr{L}(v)=-(L+a I)^{-1}\left(v I-\ell_{1} \ell_{2}\left(\ell_{3}+(v+a)\right)^{-1}\right) .
$$


Let $\Re_{a}=\{v \in \mathbb{C}: v+a \in \Re\}$. For arbitrary $v \in \mathbb{C} \backslash \Re_{a}$ the operator $\mathscr{L}(v)$ is a holomorphic operator function completely continuous in $L_{2, \mu}(\Omega)$. Moreover, the equation

$$
\hat{v}-\mathscr{L}(v) \hat{v}=0
$$

has only a trivial solution, if $\Re e v$ is sufficiently large. By the Gohberg theorem (Theorem 1.5.1 in [18]), every compact set $\mathscr{M} \subset \mathbb{C} \backslash \Re_{a}$ may contain at most a countable number of points $v$ such that the equation $(6.25)$ has a finite number of non-trivial solutions.

If $\hat{v}-\mathscr{L}(v) \hat{v}=0$, then $\lambda \hat{u}+A \hat{u}=0$ where $\hat{u}=(\hat{v}, \hat{w}), \quad \hat{w}=\left(\lambda+\ell_{3}(x)\right)^{-1}$. $\ell_{2}(x) \hat{v}(x)$. Since $\lambda \in \mathbb{C} \backslash \Re, \hat{v}(x)=0$ implies $\hat{w}(x)=0$. Hence the operator $A$ has a countable number of eigenvalues accumulating at infinity and at $\Re$.

Now we go back to the estimate (6.19). It can be derived from (6.23) and (6.20) for $\lambda \in \Sigma_{\chi, \rho}$; we show that it holds also for $\lambda \in \mathfrak{C}_{\varkappa, \beta_{1}}$ with $\chi$ so small that $\Re e \lambda=-x|\Im m \lambda|,|\lambda|=\rho$ implies $\Re e \lambda \geqslant-\beta_{1}$. By assumption (b) for sufficiently small $\varkappa$ no $\lambda$ from the compact set

$$
\overline{\mathfrak{C}_{\chi, \beta_{1}} \backslash \Sigma_{\chi, \rho}} \equiv \mathfrak{G}
$$

can be an eigenvalue of $-A$ hence for any $v=\lambda-a$ with $\lambda \in \subseteq$ equation (6.25) has only a trivial solution. By applying Fredholm alternative theorem to the operator $\mathscr{L}(v)(v=\lambda-a)$ we have, for any $\hat{f} \in X$, the existence of the solution $\hat{v}$ of problem (6.21) and (6.22) with $\lambda \in \Xi$ . Finally for any $\hat{f} \in X$ the solution of problem $(6.17)$ is then $\hat{u}=(\hat{v}, \hat{w})$ where $\hat{w}$ is given in $(6.20)$.

To prove (6.19) for $\lambda \in \mathfrak{C}_{\chi, \beta_{1}}$ it suffices to obtain a uniform estimate

$$
\|\hat{u}\|_{X} \leq c\|\hat{f}\|_{X}
$$

for all $\lambda$ from the compact set $\subseteq$.

If (6.26) is not true, then there exist sequences $\lambda_{m} \in \mathcal{\Xi}$, and $\hat{u}_{m}=\left(\hat{v}_{m}, \hat{w}_{m}\right)$ such that $\left\|\hat{u}_{m}\right\|_{X} \geq m\|\hat{f}\|_{X}$. The elements $U_{m}=\left\|\hat{u}_{m}\right\|_{X}^{-1} \hat{u}_{m}=\left(V_{m}, W_{m}\right)$ satisfy the equation $\lambda_{m} U_{m}+A U_{m}=\left\|\hat{u}_{m}\right\|_{X}^{-1} \hat{f}$, i.e.,

$$
\left\{\begin{array}{l}
v_{m}(L+a I)^{-1} V_{m}+V_{m}+(L+a I)^{-1} \ell_{1} W_{m}=\left\|\hat{u}_{m}\right\|_{X}^{-1}(L+a I)^{-1} \hat{g}, \\
\left(v_{m}+a\right) W_{m}+\ell_{2} V_{m}+\ell_{3} W_{m}=\left\|\hat{u}_{m}\right\|_{X}^{-1} \hat{h}, \\
\left.\frac{\partial V_{m}}{\partial n}\right|_{x \in \partial \Omega}=0 .
\end{array}\right.
$$

There exist $m_{k} \rightarrow+\infty$ (as $\left.k \rightarrow+\infty\right)$ such that the sequence $\lambda_{m_{k}}=v_{m_{k}}+a$ is convergent to $\lambda \in \mathfrak{S}$, the sequence $U_{m_{k}}$ is convergent weakly in $X$, the sequence $W_{m_{k}}$ is convergent strongly in $L_{2, \mu}(\Omega)$ and the sequence $(L+a I)^{-1} V_{m_{k}}$ is convergent strongly in $L_{2, \mu}(\Omega)$.

The existence of such sequences follows from the compactness of the imbedding of $W_{2}^{s}(\Omega)$ in $L_{2, \mu}(\Omega)$ and from the compactness of the operator $(L+a I)^{-1}$.

Setting $m=m_{k}$ in (6.27) we see that all the terms in the first equation (with the exception of $\left.V_{m_{k}}\right)$ are convergent strongly in $L_{2, \mu}(\Omega)$, so we can conclude that 
$V_{m_{k}}$ is also convergent strongly. Since $(L+a I)^{-1}$ is a bounded operator from $L_{2, \mu}(\Omega)$ in $W_{2, \mu}^{2}(\Omega)$, all the terms in this equation are convergent strongly in $W_{2, \mu}^{2}(\Omega)$. As a consequence, $\ell_{2} V_{m_{k}}$ is convergent strongly in $W_{2}^{s}(\Omega)$, (see Proposition 3.2) as well as $\left(v_{m_{k}}+a+\ell_{3}\right) W_{m_{k}}$ and $W_{m_{k}}$. Hence we can pass to the limit in (6.27) and obtain $\lambda U+A U=0$. Since $\lambda$ can not be an eigenvalue of $-A$, we conclude that $U=0$. But this contradicts to the fact that $\|U\|_{X}=1$ (since $U$ is a strong limit of $U_{m_{k}}$ in $X$ ). Hence the estimate (6.26) holds for $\lambda \in \mathfrak{S}$, and the resolvent estimate (6.18) holds for $\lambda \in \mathfrak{C}_{\chi, \beta_{1}}$.

This estimate implies (6.15) (see Theorem 1.3.4 in [19]), and this complete the proof of Theorem 6.2.

TheOrem 6.3. We assume all the hypotheses of Theorem 6.2. Then problem (6.1) has a unique solution $u=(v, w)$ with $v \in W_{2, \mu}^{2,1}\left(Q_{T}\right), w, \frac{\partial w}{\partial t} \in W_{2}^{s, 0}\left(Q_{T}\right)$, and the following estimate holds:

$$
\begin{aligned}
& \left\|e^{\beta t} v\right\|_{W_{2, \mu}^{2,1}\left(Q_{T}\right)}+\left\|e^{\beta t} w\right\|_{W_{2}^{s, 0}\left(Q_{T}\right)}+\left\|e^{\beta t} \frac{\partial w}{\partial t}\right\|_{W_{2}^{s, 0}\left(Q_{T}\right)} \\
& \quad \leq c\left(\left\|e^{\beta t} g\right\|_{L_{2, \mu}\left(Q_{T}\right)}+\left\|e^{\beta t} h\right\|_{W_{2}^{s, 0}\left(Q_{T}\right)}+\left\|w_{0}\right\|_{W_{2}^{s}(\Omega)}+\left\|v_{0}\right\|_{W_{2, \mu}^{1}(\Omega)}\right)
\end{aligned}
$$

with the constant $c$ independent of $T$. Here $T \leq+\infty$ and $\beta<b_{1}$ is a positive number.

Proof. Estimate (6.28) is established by the same arguments as (6.13). We go back to Theorem 6.1 and consider the problem (6.3). In view of the assumption (a), we have,

$$
\begin{gathered}
\left\|V(t) w_{0}\right\|_{W_{2}^{s}(\Omega)} \leq c e^{-b_{1} t}\left\|w_{0}\right\|_{W_{2}^{s}(\Omega)}, \\
\left\|e^{\beta t} \varphi\right\|_{W_{2}^{s, 0}\left(Q_{T}\right)} \leq\left\|e^{\beta t} h\right\|_{W_{2}^{s, 0}\left(Q_{T}\right)}+c\left\|e^{\beta t} v\right\|_{W_{2}^{s, 0}\left(Q_{T}\right)}
\end{gathered}
$$

and

$$
\begin{gathered}
\left\|e^{\beta t} w\right\|_{W_{2}^{s, 0}\left(Q_{T}\right)}+\left\|e^{\beta t} \frac{\partial w}{\partial t}\right\|_{W_{2}^{s, 0}\left(Q_{T}\right)}+\sup _{t<T} e^{\beta t}\|w(\cdot, t)\|_{W_{2}^{s}(\Omega)} \\
\leq c\left(\left\|w_{0}\right\|_{W_{2}^{s}(\Omega)}+\left\|e^{\beta t} \varphi\right\|_{W_{2}^{s, 0}\left(Q_{T}\right)}\right)
\end{gathered}
$$

with the constants independent of $T$ (these estimates are established by elementary calculations and applying the Young inequality for convolutions see e.g. [4]). Moreover, the functions $d$ and $\mathscr{I} v$ (defined in (6.8) and (6.10)) satisfy

$$
\begin{gathered}
\left\|e^{\beta t} d\right\|_{L_{2, \mu}\left(Q_{T}\right)} \leq\left\|e^{\beta t} g\right\|_{L_{2, \mu}\left(Q_{T}\right)}+c\left(\left\|w_{0}\right\|_{L_{2, \mu}(\Omega)}+\left\|e^{\beta t} h\right\|_{L_{2, \mu}\left(Q_{T}\right)}\right), \\
\left\|e^{\beta t} \mathscr{I} v\right\|_{L_{2, \mu}\left(Q_{T}\right)} \leq c\left\|e^{\beta t} v\right\|_{L_{2, \mu}\left(Q_{T}\right)} .
\end{gathered}
$$

Since the function $v_{\beta}(x, t)=e^{\beta t} v(x, t)$, where $v$ is a solution of $(6.7)$, satisfies the relations 


$$
\begin{aligned}
& \frac{\partial v_{\beta}}{\partial t}+L v_{\beta}=-e^{\beta t} \mathscr{I} v+e^{\beta t} d+\beta v_{\beta}, \\
& \left.\frac{\partial v_{\beta}}{\partial n}\right|_{x \in \partial \Omega}=0, \quad v_{\beta}(x, 0)=v_{0}(x),
\end{aligned}
$$

we have, by (5.3), (6.30)

$$
\begin{aligned}
\left\|e^{\beta t} v\right\|_{W_{2, \mu}^{2,1}\left(Q_{T}\right)} \leq c_{1}( & \left\|w_{0}\right\|_{W_{2}^{s}(\Omega)}+\left\|v_{0}\right\|_{W_{2, \mu}^{1}(\Omega)} \\
& \left.+\left\|e^{\beta t} h\right\|_{L_{2, \mu}\left(Q_{T}\right)}+\left\|e^{\beta t} g\right\|_{L_{2, \mu}\left(Q_{T}\right)}\right)+c_{2}\left\|e^{\beta t} v\right\|_{L_{2, \mu}\left(Q_{T}\right)},
\end{aligned}
$$

with constants independent of $T$.

We estimate the last term in (6.31) by inequality $(6.16)$ :

$$
\left\|e^{\beta t} v\right\|_{L_{2, \mu}\left(Q_{T}\right)} \leq\left\|e^{\beta t} u\right\|_{L_{2}(0, T ; X)} \leq c\left(\left\|u_{0}\right\|_{X}+\left\|e^{\beta t} f\right\|_{L_{2}(0, T ; X)}\right) .
$$

Estimate (6.28) follows from (6.29), (6.31), (6.32). Theorem 6.3 is proved.

REMARK 6.1. In the assumptions and notation of Theorem 6.3, by means of interpolation inequalities (see e.g. [27]), we can derive from (6.28) the following estimate that we will use in Section 7

$$
\begin{aligned}
& \left\|e^{\beta t} v\right\|_{W_{2, \mu}^{2,1}\left(Q_{T}\right)}+\sup _{t<T} e^{\beta t}\|v(\cdot, t)\|_{W_{2, \mu}^{1}(\Omega)}+\left\|e^{\beta t} w\right\|_{W_{2}^{s, 0}\left(Q_{T}\right)} \\
& \quad+\left\|e^{\beta t} \frac{\partial w}{\partial t}\right\|_{W_{2}^{s, 0}\left(Q_{T}\right)}+\sup _{t<T} e^{\beta t}\|w(\cdot, t)\|_{W_{2}^{s}(\Omega)} \\
& \leq c\left(\left\|e^{\beta t} g\right\|_{L_{2, \mu}\left(Q_{T}\right)}+\left\|e^{\beta t} h\right\|_{W_{2}^{s, 0}\left(Q_{T}\right)}+\left\|w_{0}\right\|_{W_{2}^{s}(\Omega)}+\left\|v_{0}\right\|_{W_{2, \mu}^{1}(\Omega)}\right) .
\end{aligned}
$$

with the constant $c$ independent of $T$. Here $T \leq+\infty$ and $\beta<b_{1}$ is a nonnegative number.

REMARK 6.2. Theorems 6.1, 6.2 and 6.3 hold true under more general assumptions concerning the operator $L$ (in particular we can choose a second order differential operator with smooth coefficients) as well as the Dirichlet boundary conditions but in order to focus the attention on the ideas and tools we have preferred to treat only a model problem.

\section{THE NON-LINEAR PROBLEM}

In this section, we apply Theorem 6.3 to the analysis of the non-linear problem

$$
\begin{gathered}
\frac{\partial u}{\partial t}+A\left(x, \frac{\partial}{\partial x}\right) u+\mathscr{R}(u)=0, \quad x \in \Omega \subset \mathbb{R}^{2}, t \in(0, T) \\
\left.\frac{\partial v}{\partial n}\right|_{x \in \partial \Omega}=0, \quad u(x, 0)=u_{0}(x),
\end{gathered}
$$


where $A$ is linear operator (1.2) and $\mathscr{R}(u)=(\mathscr{P}(u), \mathscr{Z}(u))$ is a vector field of nonlinear terms.

From now on we assume that $\mathscr{P}$ is a linear combination of the terms $p_{j}$, $j=1, \ldots, 7$, satisfying the following conditions $(1)-(7)$ where $m$ is a positive integer.

We denote the partial derivative $\frac{\partial v}{\partial x_{k}}$ simply by $v_{k}$, the second derivative $\frac{\partial^{2} v}{\partial x_{k}, \partial x_{l}}$ by $v_{k, l}$ and $\left(\frac{\partial^{2} v}{\partial x_{k} \partial v_{x_{l}}}\right)_{k, l=1,2}$ by $D^{2} v$.

(1) $\left|p_{1}(v)\right| \leq c|v|^{\sigma_{1}},\left|\frac{d p_{1}}{d v}\right| \leq c|v|^{\sigma_{1}-1}$ with $2 \leqslant \sigma_{1}<2+1 / \mu$,

(2) $\left|p_{2}(v, \nabla v)\right| \leq c|v|^{\tau_{1}}|\nabla v|,\left|\frac{\partial p_{2}}{\partial v}\right| \leq c|v|^{\tau_{1}-1}|\nabla v|,\left|\frac{\partial p_{2}}{\partial v_{k}}\right| \leq c|v|^{\tau_{1}}$, where $1 \leq \tau_{1}<1 / \mu$,

(3) $\left|p_{3}(v, w)\right| \leq c|w|^{m}|v|^{\sigma_{2}},\left|\frac{\partial p_{3}}{\partial v}\right| \leq c|v|^{\sigma_{2}-1}|w|^{m},\left|\frac{\partial p_{3}}{\partial w}\right| \leq c|v|^{\sigma_{2}}|w|^{m-1}$ with $2 \leq \sigma_{2}<$ $2+1 / \mu$

(4) $\left|p_{4}(v, \nabla v, w)\right| \leq c|w|^{m}|v|^{\tau_{2}}|\nabla v|, \quad\left|\frac{\partial p_{4}}{\partial v}\right| \leq c|v|^{\tau_{2}-1}|\nabla v||w|^{m}, \quad\left|\frac{\partial p_{4}}{\partial v_{k}}\right| \leq c|v|^{\tau_{2}}|w|^{m}$, $\left|\frac{\partial p_{4}}{\partial w}\right| \leq c|v|^{\tau_{2}}|\nabla v||w|^{m-1}$ with $1 \leq \tau_{2}<1 / \mu$

(5) $\left|p_{5}\left(D^{2} v, w\right)\right| \leq c|w|^{m}\left|D^{2} v\right|,\left|\frac{\partial p_{5}}{\partial v_{k, l}}\right| \leq c|w|^{m},\left|\frac{\partial p_{5}}{\partial w}\right| \leq c\left|D^{2} v\right||w|^{m-1}$,

(6) $\left|p_{6}(v, \nabla w)\right| \leq c|v|^{\sigma_{3}}|\nabla w|, \quad\left|\frac{\partial p_{6}}{\partial v}\right| \leq c|v|^{\sigma_{3}-1}|\nabla w|, \quad\left|\frac{\partial p_{6}}{\partial w_{k}}\right| \leq c|v|^{\sigma_{3}}$, with $1 \leq \sigma_{3}<$ $1+\frac{(s-1)}{\mu}$

(7) $\left|p_{7}(\nabla v, \nabla w)\right| \leq c|\nabla v||\nabla w|,\left|\frac{\partial p_{7}}{\partial v_{k}}\right| \leq c|\nabla w|,\left|\frac{\partial p_{7}}{\partial w_{k}}\right| \leq c|\nabla v|$.

These conditions are satisfied if $p_{i}$ are polynomials of degree $\geqslant 2$.

The non-linear operator $\mathcal{Q}$ is a linear combination of terms $q_{j}$ of the type

(8) $q_{8}(v, w)=w^{m} v$

(9) $q_{9}(w)=w^{m+1}$

where $m$ is a positive integer.

We refer to the Introduction and to the references cited there for a discussion of mixed type systems modeling biological phenomena, ecological studies and physical problems.

We will prove the following stability result.

THEOREM 7.1. Let the operator A, defined in (1.2) satisfy all the assumptions of Theorem 6.2 and the operator $\mathscr{R}(u)$ be a vector field of nonlinear terms satisfying the above conditions (1)-(9). Then there exists $\eta>0$ such that if

$$
\left\|u_{0}\right\|_{X}=\left\|v_{0}\right\|_{W_{2, \mu}^{1}(\Omega)}+\left\|w_{0}\right\|_{W_{2}^{s}(\Omega)} \leq \eta
$$

problem (7.1) has a unique solution $u=(v, w), v \in W_{2, \mu}^{2,1}\left(Q_{\infty}\right), w \in W_{2}^{s, 0}\left(Q_{\infty}\right)$, $\frac{\partial w}{\partial t} \in W_{2}^{s, 0}\left(Q_{\infty}\right)$, and the following estimate holds

$$
\begin{gathered}
\left\|e^{\beta t} v\right\|_{W_{2, \mu}^{2,1}\left(Q_{\infty}\right)}+\left\|e^{\beta t} w\right\|_{W_{2}^{s, 0}\left(Q_{\infty}\right)}+\left\|e^{\beta t} \frac{\partial w}{\partial t}\right\|_{W_{2}^{s, 0}\left(Q_{\infty}\right)} \\
\leq c\left(\left\|w_{0}\right\|_{W_{2}^{s}(\Omega)}+\left\|v_{0}\right\|_{W_{2, \mu}^{1}(\Omega)}\right) \leq c \eta
\end{gathered}
$$

where $0 \leqslant \beta<b_{1}$. 
We can regard (7.1) as the problem for perturbations of the zero solution of (7.1) (with $u_{0}=0$ ), and estimate (7.3) proves the exponential stability of this solution.

The proof is based on inequality (6.28) and on the estimate of the norms $\left\|e^{\beta t} \mathscr{R}(u)\right\|_{L_{2}(0,+\infty ; X)}$ and $\left\|e^{\beta t} \delta \mathscr{R}(u, U)\right\|_{L_{2}(0,+\infty ; X)}$, where

$$
\delta \mathscr{R}(u, U)=\int_{0}^{1} \frac{d}{d r} \mathscr{R}(u+r U) d r=\mathscr{R}(u+U)-\mathscr{R}(u) .
$$

We also make use of the following Lemma that can be established by elementary calculations.

LEMMA 7.2. Let $a_{h}$ be a sequence of non negative real numbers such that

$$
a_{1} \leq c_{1} \delta, \quad \text { and } \quad a_{h+1} \leq c_{1} \delta+c_{2} a_{h}^{\sigma}, \quad \sigma>1
$$

Then for any choice of $c_{2}, c_{1}$ and $\sigma>1$ there exists a positive constant $c_{3}>c_{1}$ that guarantees the uniform estimate

$$
a_{h} \leq c_{3} \delta
$$

if $\delta$ is sufficiently small.

We start with the estimates of $\left\|p_{j}\right\|_{L_{2, \mu}\left(Q_{T}\right)}$ and $\left\|q_{j}\right\|_{L_{2}\left(0, T ; W_{2}^{s}(\Omega)\right)}$.

Proposition 7.1. In the previous notation and assumptions the following estimates hold:

$$
\begin{gathered}
\sum_{j=1}^{7}\left\|e^{\beta t} p_{j}\right\|_{L_{2, \mu}\left(Q_{T}\right)} \\
\leq c\left(Y^{\sigma_{1}}+Y^{\tau_{1}+1}+Y^{m+\sigma_{2}}+Y^{m+\tau_{2}+1}+Y^{m+1}+Y^{\sigma_{3}+1}+Y^{2}\right) \\
\sum_{j=8}^{9}\left\|e^{\beta t} q_{j}\right\|_{W_{2}^{s, 0}\left(Q_{T}\right)} \leq c Y^{m+1}
\end{gathered}
$$

where $Y=Y(u, \beta)$ is the sum

$$
\begin{aligned}
Y(u, \beta)= & \left\|e^{\beta t} v\right\|_{W_{2, \mu}^{2,1}\left(Q_{T}\right)}+\sup _{t<T} e^{\beta t}\|v(\cdot, t)\|_{W_{2, \mu}^{1}(\Omega)}+\left\|e^{\beta t} w\right\|_{W_{2}^{s, 0}\left(Q_{T}\right)} \\
& +\left\|e^{\beta t} \frac{\partial w}{\partial t}\right\|_{W_{2}^{s, 0}\left(Q_{T}\right)}+\sup _{t<T} e^{\beta t}\|w(\cdot, t)\|_{W_{2}^{s}(\Omega)},
\end{aligned}
$$

$\sigma_{1}, \sigma_{2}, \sigma_{3}, \tau_{1}, \tau_{2}, m$ are the exponents in items $(1)-(9)$ and $T \leq+\infty$. 
ProOF. We estimate at first the norm of $p_{1}(v)$. By the embedding results (see Propositions 3.1 and 3.2, formulas (3.6) and (3.15)),

$$
\left\|p_{1}(v)\right\|_{L_{2, \mu}(\Omega)} \leq c \sup _{x \in \Omega}|v(x, t)|\left\||v|^{\sigma_{1}-1}\right\|_{L_{2, \mu}(\Omega)} \leq c\|v\|_{W_{2, \mu}^{2}(\Omega)}\|v\|_{W_{2, \mu}^{1}(\Omega)}^{\sigma_{1}-1}
$$

which implies

$$
\left\|e^{\beta t} p_{1}(v)\right\|_{L_{2, \mu}\left(Q_{T}\right)} \leq c \sup _{t<T} e^{\beta t\left(\sigma_{1}-1\right)}\|v(\cdot, t)\|_{W_{2, \mu}^{1}(\Omega)}^{\sigma_{1}-1}\left\|e^{\beta t} v\right\|_{W_{2, \mu}^{2,0}\left(Q_{T}\right)} \leq c Y^{\sigma_{1}}
$$

(since $\left.e^{\beta t} \geqslant 1\right)$.

Now we evaluate the norm of $p_{2}(v, \nabla v)$. By the Hölder inequality,

$$
\left\|p_{2}(v, \nabla v)\right\|_{L_{2, \mu}(\Omega)} \leq c\left(\int_{\Omega}|\nabla v|^{2 p}|x|^{2 \mu} d x\right)^{1 / 2 p}\left(\int_{\Omega}|v|^{2 \tau_{1} p^{\prime}}|x|^{2 \mu} d x\right)^{1 / 2 p^{\prime}}
$$

where $1 / p+1 / p^{\prime}=1$ and

$$
\frac{\mu}{\mu+1}<\frac{1}{p}<1-\frac{\tau_{1} \mu}{\mu+1}
$$

(since $\tau_{1}<\frac{1}{\mu}$, such $p$ can be found, and it is easily seen that $\tau_{1} p^{\prime}<\frac{\mu+1}{\mu}$ ). Hence by the embedding result (see in Proposition 3.1 estimate (3.6) with $q=2 p<2+2 / \mu$ and $\left.q=2 \tau_{1} p^{\prime}<2+2 / \mu\right)$ we have

$$
\left\|p_{2}(v, \nabla v)\right\|_{L_{2, \mu}(\Omega)} \leq c\|v\|_{W_{2, \mu}^{1}(\Omega)}^{\tau_{1}}\|v\|_{W_{2, \mu}^{2}(\Omega)}
$$

and

$$
\left\|e^{\beta t} p_{2}(v, \nabla v)\right\|_{L_{2, \mu}\left(Q_{T}\right)} \leq c \sup _{t<T} e^{\beta t \tau_{1}}\|v(\cdot, t)\|_{W_{2, \mu}^{1}(\Omega)}^{\tau_{1}}\left\|e^{\beta t} v\right\|_{W_{2, \mu}^{2,0}\left(Q_{T}\right)} \leq c Y^{1+\tau_{1}} .
$$

Now we evaluate the norm of $p_{3}(v, w)$. It is easily seen that

$$
\left\|p_{3}(v, w)\right\|_{L_{2, \mu}(\Omega)} \leq \sup _{x \in \Omega}|w(x, t)|^{m} \sup _{x \in \Omega}|v(x, t)|\left\||v|^{\sigma_{2}-1}\right\|_{L_{2, \mu}(\Omega)},
$$

hence by (3.6),

$$
\left\|p_{3}(v, w)\right\|_{L_{2, \mu}(\Omega)} \leq c\|w\|_{W_{2}^{s}(\Omega)}^{m}\|v\|_{W_{2, \mu}^{1}(\Omega)}^{\sigma_{2}-1}\|v\|_{W_{2, \mu}^{2}(\Omega)}
$$

where we have used the embedding results (see Propositions 3.1 and 3.2, estimate (3.6), with $q=2 \sigma_{2}-2, \sigma_{2}<2+2 / \mu$ and estimates (3.14) (3.15)) and

$$
\begin{aligned}
& \left\|e^{\beta t} p_{3}(v, w)\right\|_{L_{2, \mu}\left(Q_{T}\right)} \\
& \quad \leq c \sup _{t<T} e^{\beta t m}\|w\|_{W_{2}^{s}(\Omega)}^{m} \sup _{t<T} e^{\beta t\left(\sigma_{2}-1\right)}\|v\|_{W_{2, \mu}^{1}(\Omega)}^{\sigma_{2}-1}\left\|e^{\beta t} v\right\|_{W_{2, \mu}^{2,0}\left(Q_{T}\right)} \leq c Y^{m+\sigma_{2}} .
\end{aligned}
$$


The norm of $p_{4}(v, \nabla v, w)$ is estimated in the same way as $p_{2}$ :

$$
\left\|p_{4}(v, \nabla v, w)\right\|_{L_{2, \mu}(\Omega)} \leq c\|v\|_{W_{2, \mu}^{1}(\Omega)}^{\tau_{2}}\|v\|_{W_{2, \mu}^{2}(\Omega)} \sup _{x \in \Omega}|w(x, t)|^{m}
$$

and

$$
\begin{aligned}
& \left\|e^{\beta t} p_{4}(v, \nabla v, w)\right\|_{L_{2, \mu}\left(Q_{T}\right)} \\
& \quad \leq c \sup _{t<T} e^{\beta t \tau_{2}}\|v\|_{W_{2, \mu}^{1}(\Omega)}^{\tau_{2}^{2}} \sup _{t<T} e^{\beta t m}\|w\|_{W_{2}^{s}(\Omega)}^{m}\left\|e^{\beta t} v\right\|_{W_{2, \mu}^{2}\left(Q_{T}\right)} \leq c Y^{m+1+\tau_{2}} .
\end{aligned}
$$

We pass to the estimate of $p_{5}\left(D^{2} v, w\right)$ :

$$
\begin{gathered}
\left\|p_{5}\left(D^{2} v, w\right)\right\|_{L_{2, \mu}(\Omega)} \leq \sup _{x \in \Omega}|w(x, t)|^{m}\left\|\left|D^{2} v\right|\right\|_{L_{2, \mu}(\Omega)} \\
\left\|e^{\beta t} p_{5}\left(D^{2} v, w\right)\right\|_{L_{2, \mu}\left(Q_{T}\right)} \leq c \sup _{t<T} e^{\beta t m}\|w(\cdot, t)\|_{W_{2}^{s}(\Omega)}^{m}\left\|e^{\beta t} v\right\|_{W_{2, \mu}^{2}\left(Q_{T}\right)} \leq c Y^{m+1} .
\end{gathered}
$$

Now we evaluate the norm of $p_{6}(v, \nabla w)$. We have

$$
\left\|p_{6}(v, \nabla w)\right\|_{L_{2, \mu}(\Omega)} \leq\left(\int_{\Omega}|\nabla w|^{2 p} d x\right)^{1 / 2 p}\left(\int_{\Omega}|v|^{2 p^{\prime} \sigma_{3}}|x|^{2 \mu p^{\prime}} d x\right)^{1 / 2 p^{\prime}}
$$

where $p=(2-s)^{-1}$, so that the space $W_{2}^{s-1}(\Omega)$ is continuously imbedded in $L_{2 p}(\Omega)$. We also have $2 p^{\prime} \sigma_{3}<\frac{2 \sigma_{3}}{\left(\sigma_{3}-1\right) \mu}$, hence the last integral in (7.10) can be estimated by (3.7). This gives

and

$$
\left\|p_{6}(v, \nabla w)\right\|_{L_{2, \mu}(\Omega)} \leq c\|w\|_{W_{2}^{s}(\Omega)}\|v\|_{W_{2, \mu}^{1}(\Omega)}^{\sigma_{3}}
$$

$$
\left\|e^{\beta t} p_{6}\right\|_{L_{2, \mu}\left(Q_{T}\right)} \leq c\left\|e^{\beta t} w\right\|_{W_{2}^{s, 0}\left(Q_{T}\right)} \sup _{t<T} e^{\beta t \sigma_{3}}\|v(\cdot, t)\|_{W_{2, \mu}^{1, \mu}(\Omega)}^{\sigma_{3}} \leq c Y^{1+\sigma_{3}} .
$$

Now we evaluate the norm of $p_{7}(\nabla v, \nabla w)$. We apply the Hölder inequality with the same $p$ as above and obtain

$$
\begin{aligned}
\left\|p_{7}(\nabla v, \nabla w)\right\|_{L_{2, \mu}(\Omega)} & \leq\left(\int_{\Omega}|\nabla w|^{2 p} d x\right)^{1 / 2 p}\left(\int_{\Omega}|\nabla v|^{2 p^{\prime}}|x|^{2 \mu p^{\prime}} d x\right)^{1 / 2 p^{\prime}} \\
& \leq c\|w\|_{W_{2}^{s}(\Omega)}\|v\|_{W_{2, \mu}^{2}(\Omega)}, \\
\left\|e^{\beta t} p_{7}(\nabla v, \nabla w)\right\|_{L_{2, \mu}\left(Q_{T}\right)} & \leq c \sup _{t<T} e^{\beta t}\|w(\cdot, t)\|_{W_{2}^{s}(\Omega)}\left\|e^{\beta t} v\right\|_{W_{2, \mu}^{2,0}\left(Q_{T}\right)} \leq c Y^{2} .
\end{aligned}
$$

Collecting the estimates of $p_{i}$ we arrive at (7.7). It remains to estimate $q_{8}$ and $q_{9}$. By Proposition 3.2 we have

$$
\begin{aligned}
&\left\|q_{8}(v, w)\right\|_{W_{2}^{s}(\Omega)} \leq c\|w\|_{W_{2}^{s}(\Omega)}^{m}\|v\|_{W_{2}^{s}(\Omega)} \leq c\|w\|_{W_{2}^{s}(\Omega)}^{m}\|v\|_{W_{2, \mu}^{2}(\Omega)}, \\
&\left\|e^{\beta t} q_{8}(v, w)\right\|_{W_{2}^{s, 0}\left(Q_{T}\right)} \leq c \sup _{t<T} e^{\beta t m}\|w(\cdot, t)\|_{W_{2}^{s}(\Omega)}^{m}\left\|e^{\beta t} v\right\|_{W_{2, \mu}^{2,0}\left(Q_{T}\right)} \leq c Y^{m+1}, \\
&\left\|q_{9}(w)\right\|_{W_{2}^{s}(\Omega)} \leq c\|w\|_{W_{2}^{s}(\Omega)}^{m+1}, \\
&\left\|e^{\beta t} q_{9}(w)\right\|_{W_{2}^{s, 0}\left(Q_{T}\right)} \leq c Y^{m+1} .
\end{aligned}
$$


These estimates yield (7.8), hence the proposition is proved.

To evaluate the term $\delta \mathscr{R}(u, U)$ in (7.4) we have to consider the vector field $\mathscr{R}\left(u^{(r)}\right)$ where $u^{(r)}=u+r U$ and to estimate the norms of the derivatives of the functions $\mathscr{P}\left(u^{(r)}\right), \mathscr{2}\left(u^{(r)}\right)$ i.e. the functions

$$
\begin{aligned}
& V \frac{\partial p_{i}}{\partial v}, \quad i=1,2,3,4,6, \\
& \frac{\partial V}{\partial x_{k}} \frac{\partial p_{j}}{\partial v_{k}}, \quad j=2,4,7, \\
& W \frac{\partial p_{n}}{\partial w}, \quad n=3,4,5, \\
& \frac{\partial^{2} V}{\partial x_{k} \partial x_{l}} \frac{\partial p_{5}}{\partial v_{k, l}}, \quad \frac{\partial W}{\partial x_{k}} \frac{\partial p_{6}}{\partial w_{k}}, \quad \frac{\partial W}{\partial x_{k}} \frac{\partial p_{7}}{\partial w_{k}},
\end{aligned}
$$

as well as

$$
V \frac{\partial q_{8}}{\partial v}, \quad W \frac{\partial q_{8}}{\partial w}, \quad W \frac{\partial q_{9}}{\partial w}
$$

for large and small $T$ where the terms $p_{j}=p_{j}\left(u^{(r)}\right), j=1, \ldots, 7$ and $q_{i}\left(u^{(r)}\right)$, $i=8,9$. Concerning the vector $U=(V, W)$ we assume that it has finite norm (7.9) (denoted by $Z=Z(U, \beta)$ if $\beta>0$ and by $Z_{0}$ if $\beta=0$ ) and in addition $\left.U\right|_{t=0}=\left(\left.V\right|_{t=0},\left.W\right|_{t=0}\right)=(0,0)$.

Proposition 7.2. In the previous assumptions and notation there hold the inequalities

$$
\begin{array}{rl}
\sum_{i} \| e^{\beta t} & V \frac{\partial p_{i}}{\partial v}\left\|_{L_{2, \mu}\left(Q_{T}\right)}+\sum_{j}\right\| e^{\beta t} \frac{\partial V}{\partial x_{k}} \frac{\partial p_{j}}{\partial v_{k}}\left\|_{L_{2, \mu}\left(Q_{T}\right)}+\sum_{n}\right\| e^{\beta t} W \frac{\partial p_{n}}{\partial w} \|_{L_{2, \mu}\left(Q_{T}\right)} \\
+ & \left\|e^{\beta t} \frac{\partial^{2} V}{\partial x_{k} \partial x_{l}} \frac{\partial p_{5}}{\partial v_{k, l}}\right\|\left\|_{L_{2, \mu}\left(Q_{T}\right)}+\right\| e^{\beta t} \frac{\partial W}{\partial x_{k}} \frac{\partial p_{6}}{\partial w_{k}} \|_{L_{2, \mu}\left(Q_{T}\right)} \\
+ & \left\|e^{\beta t} \frac{\partial W}{\partial x_{k}} \frac{\partial p_{7}}{\partial w_{k}}\right\|_{L_{2, \mu}\left(Q_{T}\right)}+\left\|e^{\beta t} V \frac{\partial q_{8}}{\partial v}\right\|_{W_{2}^{s, 0}\left(Q_{T}\right)} \\
+ & \left\|e^{\beta t} W \frac{\partial q_{8}}{\partial w}\right\|_{W_{2}^{s, 0}\left(Q_{T}\right)}+\left\|e^{\beta t} W \frac{\partial q_{9}}{\partial w}\right\|_{W_{2}^{s, 0}\left(Q_{T}\right)} \\
\leq & c Z\left(Y^{\sigma_{1}-1}\left(u^{(r)}, \beta\right)+Y^{\tau_{1}}\left(u^{(r)}, \beta\right)+Y^{m+\sigma_{2}-1}\left(u^{(r)}, \beta\right)+Y^{m+\tau_{2}}\left(u^{(r)}, \beta\right)\right. \\
& \left.+Y^{\sigma_{3}}\left(u^{(r)}, \beta\right)+Y\left(u^{(r)}, \beta\right)+Y^{m}\left(u^{(r)}, \beta\right)\right) .
\end{array}
$$

Moreover, if norm $Y_{0}=Y\left(u^{(r)}, 0\right) \leq M$, (see (7.9) with $\left.\beta=0\right)$ and $\left\|u_{r}(0)\right\|_{X} \leq c \eta($ see $(7.2))$ then 


$$
\begin{aligned}
\sum_{i} \| V & \frac{\partial p_{i}}{\partial v}\|\|_{L_{2, \mu}\left(Q_{T}\right)}+\sum_{j}\left\|\frac{\partial V}{\partial x_{k}} \frac{\partial p_{j}}{\partial v_{k}}\right\|_{L_{2, \mu}\left(Q_{T}\right)}+\sum_{n}\left\|W \frac{\partial p_{n}}{\partial w}\right\|_{L_{2, \mu}\left(Q_{T}\right)} \\
& +\left\|\frac{\partial^{2} V}{\partial x_{k} \partial x_{l}} \frac{\partial p_{5}}{\partial v_{k, l}}\right\|_{L_{2, \mu}\left(Q_{T}\right)}+\left\|\frac{\partial W}{\partial x_{k}} \frac{\partial p_{6}}{\partial w_{k}}\right\|_{L_{2, \mu}\left(Q_{T}\right)}+\left\|\frac{\partial W}{\partial x_{k}} \frac{\partial p_{7}}{\partial w_{k}}\right\|_{L_{2, \mu}\left(Q_{T}\right)} \\
& +\left\|V \frac{\partial q_{8}}{\partial v}\right\|_{W_{2}^{s, 0}\left(Q_{T}\right)}+\left\|W \frac{\partial q_{8}}{\partial w}\right\|_{W_{2}^{s, 0}\left(Q_{T}\right)}+\left\|W \frac{\partial q_{9}}{\partial w}\right\|_{W_{2}^{s, 0}\left(Q_{T}\right)} \\
\leq & c_{1}(M) c_{2}(\epsilon, \eta, T) Z_{0},
\end{aligned}
$$

where $\epsilon$ is a small positive number and $c_{2}(\epsilon, \eta, T)$ can be made arbitrarily small by the choice of small $\epsilon, \eta$ and $T$.

Proof. Inequality (7.13) is established in the same way as (7.7), (7.8), for instance,

$$
\begin{aligned}
\left\|V \frac{d p_{1}\left(v^{(r)}\right)}{d v^{(r)}}\right\|_{L_{2, \mu}(\Omega)} & \leq c \sup _{x \in \Omega}|V(x, t)|\left\|\left|v^{(r)}\right|^{\sigma_{1}-1}\right\|_{L_{2, \mu}(\Omega)} \\
& \leq c\|V\|_{W_{2, \mu}^{2}(\Omega)}\left\|v^{(r)}\right\|_{L_{2, \mu}(\Omega)}^{\sigma_{1}-1}, \\
\left\|e^{\beta t} V \frac{d p_{1}\left(v^{(r)}\right)}{d v^{(r)}}\right\|_{L_{2, \mu}\left(Q_{T}\right)} & \leq c \sup _{t<T} e^{\beta t\left(\sigma_{1}-1\right)}\left\|v^{(r)}(\cdot, t)\right\|_{W_{2, \mu}(\Omega)}^{\sigma_{1}-1}\left\|e^{\beta t} V\right\|_{W_{2, \mu}^{2,0}\left(Q_{T}\right)} \\
\leq & c Z Y^{\sigma_{1}-1}\left(u^{(r)}\right) .
\end{aligned}
$$

We omit the estimates of other terms.

To obtain a small constant in (7.14), we use the interpolation inequalities, for instance,

$$
\sup _{x \in \Omega}|V(x, t)| \leq c\|V\|_{W_{2}^{s}(\Omega)} \leq \epsilon\|V\|_{W_{2, \mu}^{2}(\Omega)}+c(\epsilon)\|V\|_{L_{2, \mu}(\Omega)} .
$$

This inequality follows from (3.25); it implies

$$
\begin{aligned}
\left(\int_{0}^{T} \sup _{x \in \Omega}|V(x, t)|^{2} d t\right)^{1 / 2} & \leq \epsilon\|V\|_{W_{2, \mu}^{2,0}\left(Q_{T}\right)}+c(\epsilon)\|V\|_{L_{2, \mu}\left(Q_{T}\right)} \\
& \leq \epsilon\|V\|_{W_{2, \mu}^{2,0}\left(Q_{T}\right)}+c(\epsilon) T\left\|\frac{\partial V}{\partial t}\right\|_{L_{2, \mu}\left(Q_{T}\right)} \leq(\epsilon+c(\epsilon) T) Z_{0} .
\end{aligned}
$$

Hence

$$
\left\|V \frac{\partial p_{1}}{\partial v}\right\|_{L_{2, \mu}\left(Q_{T}\right)} \leq c(M)(\epsilon+c(\epsilon) T) Z_{0} .
$$


Taking at first $\epsilon$ and then $T$ sufficiently small, we can make $\epsilon+c(\epsilon) T$ as small as necessary. The terms with the derivatives of $p_{2}$ are evaluated in the same manner with the help of estimates (3.11), (3.12). The terms with the derivatives of $p_{3}, p_{4}$ and $p_{5}$ with respect to $w$ are evaluated in the same manner with the help of the estimate

$$
\sup _{Q_{T}}|W(x, t)| \leq c \sup _{t<T}\|W(\cdot, t)\|_{W_{2}^{s}(\Omega)} \leq c \sqrt{T}\left\|\frac{\partial W}{\partial t}\right\|_{W_{2}^{s, 0}\left(Q_{T}\right)} .
$$

Now we turn to the estimate of $\frac{\partial^{2} V}{\partial x_{k} \partial x_{l}} \frac{\partial p_{5}}{\partial v_{k, l}}$. We have

$$
\left\|\frac{\partial^{2} V}{\partial x_{k} \partial x_{l}} \frac{\partial p_{5}}{\partial v_{k, l}}\right\|_{L_{2, \mu}\left(Q_{T}\right)} \leq c \sup _{t<T}\|w(\cdot, t)\|_{W_{2}^{s}(\Omega)}^{m}\|V\|_{W_{2, \mu}^{2,0}\left(Q_{T}\right)} .
$$

We use the inequality

$$
\sup _{t<T}\|w(\cdot, t)\|_{W_{2}^{s}(\Omega)} \leq\left\|w_{0}\right\|_{W_{2}^{s}(\Omega)}+\sqrt{T}\left\|\frac{\partial w}{\partial t}\right\|_{W_{2}^{s, 0}\left(Q_{T}\right)},
$$

which implies

$$
\left\|\frac{\partial^{2} V}{\partial x_{k} \partial x_{l}} \frac{\partial p_{5}}{\partial v_{k, l}}\right\|_{L_{2, \mu}\left(Q_{T}\right)} \leq c\left(\eta+\sqrt{T} Y_{0}\right)^{m} Z_{0} .
$$

We omit further details.

Proof of TheOrem 7.1. The solution of (7.1) can be constructed by successive approximations. We define $u_{1}$ as the solution of the linear problem

$$
\begin{gathered}
\frac{\partial u_{1}}{\partial t}+A\left(x, \frac{\partial}{\partial x}\right) u_{1}=0, \quad x \in \Omega, t \in(0, T), \\
\left.\frac{\partial v_{1}}{\partial n}\right|_{x \in \partial \Omega}=0, \quad u_{1}(x, 0)=u_{0}(x)
\end{gathered}
$$

and we find $u_{h}, h \geq 1$ from

$$
\begin{aligned}
& \frac{\partial u_{h+1}}{\partial t}+A\left(x, \frac{\partial}{\partial x}\right) u_{h+1}=-\mathscr{R}\left(u_{h}\right), \quad x \in \Omega, t \in(0, T), \\
& \left.\frac{\partial v_{h+1}}{\partial n}\right|_{x \in \partial \Omega}=0, \quad u_{h+1}(x, 0)=u_{0}(x) .
\end{aligned}
$$

By Proposition 7.1 if $Y\left(u_{h}\right)$ is finite, then $\mathscr{R}\left(u_{h}\right) \in L_{2}(0, T ; X)$ and the problem (7.18) is solvable. In view of Theorem 6.3 it is clear that the functions $u_{h}$ are defined for all $h$ and that the sequence $Y_{h}=Y\left(u_{h}\right)$ (see (7.9)) satisfies the conditions

$$
\begin{gathered}
Y_{1} \leq c \eta, \\
Y_{h+1} \leq c\left(Y_{h}^{2}+Y_{h}^{\sigma_{1}}+Y_{h}^{\tau_{1}+1}+Y_{h}^{m+\sigma_{2}}+Y_{h}^{m+\tau_{2}+1}+Y_{h}^{m+1}+Y_{h}^{\sigma_{3}+1}\right)+c \eta,
\end{gathered}
$$


where

$$
\eta=\left\|v_{0}\right\|_{W_{2, \mu}^{1}(\Omega)}+\left\|w_{0}\right\|_{W_{2}^{s}(\Omega)} .
$$

If $\eta$ is small, then by Lemma $7.2(7.19)$ guarantees a uniform estimate

$$
Y_{h} \leq c \eta
$$

To prove the convergence of the sequence $u_{m}$, we consider the problem for the differences $U_{h+1}=u_{h+1}-u_{h}, h \geq 2$ :

$$
\begin{gathered}
\frac{\partial U_{h+1}}{\partial t}+A\left(x, \frac{\partial}{\partial x}\right) U_{h+1}=-\delta \mathscr{R}\left(u_{h-1}, U_{h}\right), \quad x \in \Omega \subset \mathbb{R}^{2}, t \in(0, T) \\
\left.\frac{\partial V_{h+1}}{\partial n}\right|_{x \in \partial \Omega}=0, \quad U_{h+1}(x, 0)=0 .
\end{gathered}
$$

In view of Theorem 6.3 (see also Remark 6.1)

$$
Y\left(U_{h+1}\right) \leq c\left\|e^{\beta t} \delta \mathscr{R}\left(u_{h-1}, U_{h}\right)\right\|_{L_{2}(0,+\infty ; X)} .
$$

It is easily verified that $\delta \mathscr{R}\left(u_{h-1}, U_{h}\right)$ is the integral with respect to $r \in[0,1]$ of the functions (7.11) and (7.12) with $U=(V, W)=U_{h}, u=(v, w)$ and $u_{h-1}+r U_{h} \equiv u_{h}^{(r)}$. By (7.20),

$$
Y\left(u_{h}^{(r)}\right) \leq r Y\left(u_{h}\right)+(1-r) Y\left(u_{h-1}\right) \leq c \eta
$$

and by (7.21) and (7.13),

$$
Y\left(U_{h+1}\right) \leq c \eta^{\gamma} Y\left(U_{h}\right)
$$

with some $\gamma>0$. Hence

$$
\sum_{h=2}^{K} Y\left(U_{h+1}\right) \leq c \eta^{\gamma} \sum_{h=2}^{K} Y\left(U_{h}\right) .
$$

If $c \eta^{\gamma}<1$, then we can conclude from this estimate that the sum $\sum_{h=2}^{K} Y\left(U_{h}\right)$ is uniformly (with respect to $K$ ) bounded, and the sequence $u_{h}$ is convergent strongly in the norm $Y$ to the solution of the problem (7.1). In view of (7.20), the solution satisfies (7.3).

Now we prove the uniqueness of the solution. Assume that along with the solution $u=(v, w)$ constructed above there exists another solution $u^{\prime}$ with finite norm $Y_{0}\left(u^{\prime}\right) \equiv Y^{\prime}$ in the interval $t \in\left(0, T_{0}\right)$ (this norm is defined by (7.9) with $\beta=0)$. We consider the problem for the difference $U=u^{\prime}-u$

$$
\begin{gathered}
U+A U=-\delta \mathscr{R}(u, U), \quad x \in \Omega \subset \mathbb{R}^{2}, t \in(0, T) \\
\left.\frac{\partial V}{\partial n}\right|_{x \in \partial \Omega}=0, \quad U(x, 0)=0 .
\end{gathered}
$$


We observe that the norm $Y_{0}(u+r U), r \in[0,1]$, is bounded by a certain constant $B$ in the interval $t \in\left[0, T_{0}\right]$ (see (7.22)). By (6.33) and (7.14), (see also (7.21)) for arbitrary $T \leq T_{0}$

$$
Y_{0, T}(U) \leq c_{1}(B) c_{2}(\epsilon, \eta, T) Y_{0, T}(U),
$$

where $Y_{0, T}(U)$ is the norm of $U$ in the interval $(0, T)$ and $c_{1}(B)$ is a constant dependent only on $B$. We proceed as previously and choosing $\epsilon$ and $T$ in an appropriate way we may make the constant $c_{1}(B) c_{2}(\epsilon, \eta, T)$ less that 1 . It follows that $Y_{0, T}(U)=0$, i.e., $u^{\prime}=u$ for $t<T$. By a finite number of steps we can show that $u^{\prime}=u$ for $t<T_{0}$. This concludes the proof of the theorem.

REMARK 7.1. Theorem 7.1 holds true under more general assumptions concerning the operators $\mathscr{P}=\sum_{i} c_{i}\left(\mathscr{P}_{i}\left(v, \nabla v, D^{2} v, w\right)\right)$ and $\mathscr{Z}=\sum_{k} c_{k}\left(\mathscr{Q}_{k}(v, w)\right)$ where $\mathscr{P}_{i}, \mathscr{Q}_{k}$ are of the type described in items from (1) to (7) and (8)(9) (respectively) with respect to their arguments. In particular we may assume that the functions $\mathscr{P}_{i}, \mathscr{Q}_{k}$ have coefficients belonging to the space $W_{2}^{s}(\Omega)$ with respect to the space variable $x$ and independent of $t$.

\section{REFERENCES}

[1] Adams R. A.: Sobolev Spaces, New York: Academic Press, 1975.

[2] Agranovich M. S. - Vishik M. I.: Elliptic problems with a parameter and parabolic problems of general type, Russian Math. Surveys. (1964), 53-157.

[3] Baвich V. M.: On the problem of extension of functions, Uspekhi Mat. Nauk (Russian Math. Surweys) 8, (2) (1953), 111-113.

[4] Beckner W.: Inequalities in Fourier Analysis, Annals of Mathematics 102 (1975), 159-183.

[5] Belonosov V. S.: Estimates of the solutions of parabolic systems in weighted Hölder classes and some their applications, Adv. Math. USSR Sbornik 110, (152)(1979), $163-188$.

[6] Belonosov V. S. - Višnevskiř M. P.: On the stability of stationary solutions of nonlinear parabolic systems, Adv. Math. USSR Sbornik 33, (4)(1977), 465-484.

[7] Borsuk M. - Kondratiev V.: Elliptic Boundary Value Problems of Second Order in Piecewise Smooth Domains, North-Holland Mathematical Library Elsevier 2006.

[8] Chunn L. H. - Yagi A.: Dynamical systems for forest kinematic model, Math. Sci. Appl. 16, (2) (2006), 393-409.

[9] Chunn L. H. - Tsujikawa T. - Yagi A.: Asymptotic behavior of solutions for forest kinematic model, Funkcial Ekvac. 49, (3) (2006), 427-449.

[10] Chunn L. H. - Tsujikawa T. - Yagi A.: Stationary solutions for forest kinematic model, Glasg. Math. J. 51 (2009), 1-17.

[11] Frolova E. V.: On a certain nonstationary problem in a dihedral angle I, J. Soviet Math. 70 (1994), 1828-1840.

[12] Frolova E. V. - Solonnikov V. A.: On a certain nonstationary problem in a dihedral angle II, 70 (1994), 1841-1846.

[13] Garroni M. G. - Solonnikov V. A. - Vivaldi M. A.: On the oblique derivative problem in an infinite angle, Topological Methods in Nonlinear Analysis 7 (1996), 299-325. 
[14] Garroni M. G. - Solonnikov V. A. - Vivaldi M. A.: Existence and regularity results for oblique derivatives problems for heat equations in an angle, Proceedings of the Royal Society of Edinburgh 128 A (1998), 47-79.

[15] Garroni M. G. - Solonnikov V. A. - Vivaldi M. A.: Green Function for the Heat Equation with Oblique Boundary Conditions in an Angle, Annali della Scuola Normale di Pisa 27 (4) (1998), 455-485.

[16] Garroni M. G. - Solonnikov V. A. - Vivaldi M. A.: The Exponential Behaviour of Green Function in a Dihedral Angle, Communications in Contemporary Mathematics 3 (4) (2001), 571-592.

[17] Garroni M. G. - Solonnikov V. A. - Vivaldi M. A.: Schauder estimates for a system of equations of mixed type, Rendiconti di Matematica VII 29 (2009), 117-132.

[18] Gohberg I. C. - Krejn M. G.: Intruduction à la théorie des opérateurs linéaires non auto-adjoints dans un espace hilbertien, Monographies Universitaires de Mathématiques (1971), DUNOD Paris.

[19] Henry D.: Geometric theory of semilinear parabolic equations, Lectures Notes Vol. 840, Spriger New York, 1981.

[20] Hestenes M.: Extension of the range of a differentiable function, Duke Math. J., 8 (1941), 183-192.

[21] Kondrat'iev V. A.: Boundary-value problems for elliptic equations in domains with conical or angular point, Trans. Moscow Math. J. 1967, (1968), 227-314.

[22] Kozlov V. A. - Maz'ya V. G. - Rossmann J.: Elliptic Boundary Value Problems in Domains with Point Singularities, Mathematical Surveys and Monographs 52, American Mathematical Society, 1997.

[23] Kozlov V. A.: On coefficients in the asymptotics of solutions of initial-boundary parabolic problems in domains with a conical point, Siberian Math. J 29, (1988), 227-314.

[24] Kozlov V. A.: On the asymptotics of the Green function and the Poisson kernels for the mixed parabolic problems in a cone I and II, Zeithschrift für Analysis und ihure Andwendungen 8, (1989), 131-151 and 10 (1991), 27-42.

[25] Kutznetsov Yu A. - Antonovsky M. Ya - Biktashev V. N. - Aponima A.: A crossdiffusion model of forest boundary dynamics, J Math. Biol., 32 (1994), 219-232.

[26] Jones D. S. - Sleeman B. D.: Differential Equations and mathematical biology, Allen and Unwin, London, 1983.

[27] Lions J. L. - Magenes E.: Problèmes aux limites non homogènes et applications, 1Travaux et Recherches Mathématiques, No. 17 Dunod, Paris 1968.

[28] Mulone G. - Solonnikov V. A.: Linearization principle for the system of equation of a mixed type, Nonlinear Analysis 71 (2009), 1019-1031.

[29] Murray J. D.: Mathematical biology I. An introduction, Third edition. Interdisciplinary Applied Mathematics 17, Springer-Verlag, New York 2002.

[30] Murray J. D.: Mathematical biology II. Spatial models and biomedical applications, Third edition. Interdisciplinary Applied Mathematics 18, Springer-Verlag, New York 2003.

[31] Nazarov A.: L L-estimates for a solution to Dirichlet problem and Neuman prpblem for the heat equation in an wedge of arbitrary codimension, J. Math. Sci. h 106 (2001), 2989-3014.

[32] Pao C. V.: Nonlinear parabolic and elliptic equations, Plenum Press, New York 1992.

[33] Paley R. A. C. - Wiener N.: Fourier transforms in the complex domain, American Mathematical Society Colloquium Publications 19 American Mathematical Society Providence 1987. 
[34] Sмітн K. T.: Inequalities for formally positive integro-differential forms, Bull. Amer. Math. Soc. 67 (1961), 368-370.

[35] Solonnikov V. A.: Solvability of the classical initial-boundary-value problems for the heat-conduction in a dihedral angle, J. Sov. Math. 32 (1986), 526-546.

[36] Solonnikov V. A.: On boundary value problems for linear parabolic systems of differential equations of general form, Proc. Math. Inst. Steklov, 83 (1965), 3-162.

[37] Whitney H.: Analytic extensions of differentiable functions defined in closed sets, Trans. Amer. Math. Soc. 36, (1934), 63-89.

[38] WU Y. - LIN Y.: The stability of steady states for a model with diffusion and spatial average, J. Math. Anal. Appl. 232 (1999), 259-271.

[39] Zaionchkovski V. - Solonnikov V. A.: On Neumann problem for second order elliptic equations in domains with edges on the boundary, J. Sov. Math. 27 (2) (1984), 2561-2586.

Received 1 June 2012,

and in revised form 7 September 2012.

V. A. Solonnikov

Petersburg Departement of Steklov Institute of Mathematics

27 Fontanka, 191023, St. Petersburg

Russia

solonnik@pdmi.ras.ru

M. A. Vivaldi

Dipartimento di Scienze di Base e Applicate per l'Ingegneria

Sapienza Università di Roma

Via A. Scarpa 16, 00161 Roma

Italy

maria.vivaldi@sbai.uniroma1.it 
